Review Article

\title{
On Different Types of Single-Valued Neutrosophic Covering Rough Set with Application in Decision-Making
}

\author{
Xiongwei Zhang, ${ }^{1}$ Mohammed Atef $\mathbb{D},{ }^{2}$ and Ahmed Mostafa Khalil $\mathbb{D}^{3}$ \\ ${ }^{1}$ School of Mathematics and Statistics, Yulin University, Yulin 719000, China \\ ${ }^{2}$ Department of Mathematics and Computer Science, Faculty of Science, Menoufia University, Menoufia, Egypt \\ ${ }^{3}$ Department of Mathematics, Faculty of Science, Al-Azhar University, Assiut 71524, Egypt \\ Correspondence should be addressed to Ahmed Mostafa Khalil; a.khalil@azhar.edu.eg
}

Received 27 May 2021; Revised 7 July 2021; Accepted 15 July 2021; Published 31 July 2021

Academic Editor: Lazim Abdullah

Copyright (c) 2021 Xiongwei Zhang et al. This is an open access article distributed under the Creative Commons Attribution License, which permits unrestricted use, distribution, and reproduction in any medium, provided the original work is properly cited.

\begin{abstract}
This paper aims to propose the notion of Type- 1 single-valued neutrosophic complementary $\beta$-neighborhood (briefly, Type- 1 SVN complementary $\beta$-neighborhood) and use it to introduce a novel class of 1 -single-valued neutrosophic $\beta$-covering rough set (briefly, 1-SVN $\beta$-CRS). Then, we will merge the 1-SVN $\beta$-neighborhood and 1-SVN complementary $\beta$-neighborhood to create new two models of 1-SVN $\beta$-CRS. Furthermore, we will discuss the relationships between the present work and Wang and Zhang's work. For further study on Type-2 Wang and Zhang's models, we will define the 2-SVN complementary $\beta$-neighborhood and use it to present a novel class of 2-SVN $\beta$-CRS. Also, we combine the 2 -SVN $\beta$-neighborhood and 2 -SVN complementary $\beta$-neighborhood to investigate the new two models of 2-SVN $\beta$-CRS. Lately, we will demonstrate two illustrative examples as real problems to show the differences between two of our approaches and Wang and Zhang's approach.
\end{abstract}

\section{Introduction}

In 1982, the world-known new notion called rough sets (briefly, RSs) dealt with uncertain data on the hand of Pawlak $[1,2]$. This notion helped researchers in several areas of research to develop these areas through RS, for instance, there are many published papers (see [3-19]). The famed generalization of RS is covering rough sets (briefly, CRSs). The CRSs were studied by many specialists from different views which made an evolution in many fields such as computer science, mathematics, and chemistry. Some of the relevant studies helped scholars to solve many life problems [20-33]. Consequently, in 1990, the notions of fuzzy rough sets (briefly, FRSs) and rough fuzzy sets (briefly, RFSs) are defined by Dubois and Prade [34] from the merging between the CRS and the fuzzy sets (briefly, FSs) which appeared by Zadeh [35]. From this point of view, the new kinds of covering fuzzy rough sets (briefly, CFRSs) through the fuzzy $\beta$-neighborhoods were called fuzzy $\beta$ covering rough sets (briefly, F $\beta$ CRSs) (see [36]). To complete this study, Yang et al. $[37,38]$ defined several basic notions of fuzzy complementary $\beta$-neighborhoods, fuzzy $\beta$-minimal description, and fuzzy $\beta$-maximal description to establish new classes of $\mathrm{F} \beta \mathrm{CRS}$.

The notion of single-valued neutrosophic sets (briefly, SVNS) was developed by Wang et al. [39]. SVNS is a natural extension of the intuitionistic fuzzy set (briefly, IFS) [40]. Smarandache [41] investigated a new set called neutrosophic set as a generalization of mathematical tools (i.e., fuzzy set [35], interval-valued fuzzy set [42], IFS [40], and interval-valued intuitionistic fuzzy set [43]). In 2015, Mondal and Pramanik [44] demonstrated a new terminology called rough neutrosophic set. By using SVN relation, Yang et al. [45] introduced the SVN rough set model, and based on the notion of Type-1 SVN $\beta$-neighborhoods, Wang and Zhange [46] proposed two models of Type-1 SVN $\beta$-covering rough sets (briefly, SVN $\beta$-CRS). Furthermore, they presented a new kind of SVN $\beta$-CRS called Type- 2 SVN $\beta$-CRS utilizing Type- 2 SVN $\beta$-neighborhoods in [47]. The notions of 
neutrosophic soft rough sets and its generalizations are presented in [48-53].

By the above discussion and extend the other work (see $[46,47])$ in SVN $\beta$-CRS. We will generalize these methods in $[46,47]$ by boosting the lower approximation and minimizing the upper approximation, which is a big challenge to every author. Consequently, the motivation of this paper is to improve this area is obtained by introducing the notion of 1-SVN complementary $\beta$-neighborhood (resp., 2-SVN complementary $\beta$-neighborhood) to build novel classes of 1SVN $\beta$-CRS (resp., 2 -SVN $\beta$-CRS). And, by joining 1 -SVN $\beta$-neighborhoods (resp., 2-SVN $\beta$-neighborhoods) and 1SVN complementary $\beta$-neighborhood (resp., 2-SVN complementary $\beta$-neighborhood), we obtain two new SVN $\beta$-neighborhoods which establish two new models of 1 SVN $\beta$-CRS (resp., 2-SVN $\beta$-CRS). Also, we discuss the properties of the two proposed covering methods. Finally, we apply our work (i.e., two proposed methods) to solve decision-making problems.

The organization of this article is as follows. In Section 2, we give a basic notion about the presented study. Section 3 establishes the definition of 1-SVN complementary $\beta$-neighborhood, and hence, a new model of 1 -SVN $\beta$-CRS is proposed. Also, by merging between the 1-SVN $\beta$-neighborhoods and its complementary, we set up two other models of 1 -SVN $\beta$-CRS. Thus, the relevant characteristics are also studied. Section 4 constructs the notion of 2 -SVN complementary $\beta$-neighborhood, and thus, a new model of 2 -SVN $\beta$-CRS is proposed. By merging between the 2-SVN $\beta$-neighborhoods and its complementary, we also set up two other models of 2-SVN $\beta$-CRS. Then, the relevant properties are also discussed. The decision-making approaches to the two methods are mentioned in Sections 3 and 4 are investigated in Section 5. Also, in this section, we compare between our approach and Wang and Zhang's approach. Section 6 shows the overall benefits of our study.

\section{Preliminaries}

In this section, we review some basic terminologies about the subject of this study.

Definition 1 (Cf. [26]). Assume that $\Omega$ is a universe and $\widetilde{\Gamma}$ is a family of subsets of $\Omega$. If no element in $\widetilde{\Gamma}$ is empty and $\Omega=U_{\widetilde{C} \in \widetilde{\Gamma}} \widetilde{C}$, then $\widetilde{\Gamma}$ is called a covering of $\Omega$, and $(\Omega, \widetilde{\Gamma})$ is called a covering approximation space (briefly, CAS).

Definition 2 (Cf. [54, 55]). Assume that $\Omega$ is a universe. We say $\widetilde{\Gamma}=\left\{\widetilde{C}_{1}, \widetilde{C}_{2}, \ldots, \widetilde{C}_{m}\right\}$, with $\widetilde{C}_{i} \in \mathscr{F}(\Omega)(i=1,2, \ldots, m)$, a fuzzy covering (briefly, FC) of $\Omega$ if $\left(\cup_{i=1}^{m} \widetilde{C}_{i}\right)(x)=1$, for each $x \in \Omega$.

The notion of fuzzy $\beta$-covering was discovered by $\mathrm{Ma}$ [36] $(0<\beta \leq 1)$. This notion is considered as a generalization of $\quad$ FC. If $\quad \widetilde{\Gamma}=\left\{\widetilde{C}_{1}, \widetilde{C}_{2}, \ldots, \widetilde{C}_{m}\right\}$, with $\widetilde{C}_{i} \in \mathscr{F}(\Omega)(i=1,2, \ldots, m)$, then $(\Omega, \widetilde{\Gamma})$ is called a fuzzy $\beta$-covering approximation space (briefly, F $\beta$ CAS).

Definition 3 (Cf. [54, 55]). Assume that $\Omega$ is not an empty set. For each $x \in \Omega$, define the SVN set $\mathscr{A} \subseteq \Omega$ as the following formula:

$$
\mathscr{A}=\left\{\left\langle x, \mathscr{T}_{\mathscr{A}}(x), \mathscr{I}_{\mathscr{A}}(x), \mathscr{F}_{\mathscr{A}}(x)\right\rangle\right\} .
$$

where $\mathscr{T}_{\mathscr{A}}: \Omega \longrightarrow[0,1]$ is the degree of truth membership of the element $x$ to $\mathscr{A}, \mathscr{I}_{\mathscr{A}}: \Omega \longrightarrow[0,1]$ is the degree of indeterminacy membership of the element $x$ to $\mathscr{A}$, and $\mathscr{F}_{\mathscr{A}}: \Omega \longrightarrow[0,1]$ is the degree of falsity membership. These variables satisfy $0 \leq \mathscr{T}_{\mathscr{A}}+\mathscr{I}_{\mathscr{A}}+\mathscr{F}_{\mathscr{A}} \leq 3$.

In 2018, Wang et al. [39] established the notion of SVN $\beta$-covering approximation space, and then, Wang and Zhang $[46,47]$ used this notion to create two types of the covering method as in the following definition.

Definition 4 (Cf. [46, 47]). Let $\Omega$ be a universe and SVN $(\Omega)$ be the SVN power set of $\Omega$. For a SVN number $\beta=(a, b, c)$, we call $\widetilde{\Gamma}=\left\{\widetilde{C}_{1}, \widetilde{C}_{2}, \ldots, \widetilde{C}_{m}\right\}$, with $\widetilde{C}_{i} \in \operatorname{SVN}(\Omega)$ $(i=1,2, \ldots, m)$, a Type-1 SVN $\beta$-covering (a Type-2 SVN $\beta$-covering) of $\Omega$ if $\widetilde{C}_{i}(x) \geq \beta\left(\widetilde{C}_{i}(x) \geqslant \beta\right)$, for each $x \in \Omega$. Moreover, $(\Omega, \widetilde{\Gamma})$ is called a Type-1 SVN $\beta$-covering approximation space (a Type-2 SVN $\beta$-covering approximation space) (briefly, 1-SVN $\beta$ CAS (2-SVN $\beta$ CAS)).

If $\mathscr{A}=\left\langle a_{1}, b_{1}, c_{1}\right\rangle$ and $\mathscr{B}=\left\langle a_{2}, b_{2}, c_{2}\right\rangle$ are two SVN numbers, then

(i) $\mathscr{A} \leq \mathscr{B} \Leftrightarrow a_{1} \leq a_{2}, b_{1} \geq b_{2}, c_{1} \geq c_{2}$

(ii) $\mathscr{A} \geq \mathscr{B} \Leftrightarrow a_{1} \geq a_{2}, b_{1} \leq b_{2}, c_{1} \leq c_{2}$

(iii) $\mathscr{A} \prec \mathscr{B} \Leftrightarrow a_{1} \leq a_{2}, b_{1} \leq b_{2}, c_{1} \geq c_{2}$

(iv) $\mathscr{A} \geqslant \mathscr{B} \Leftrightarrow a_{1} \geq a_{2}, b_{1} \geq b_{2}, c_{1} \leq c_{2}$

Here, $\forall \mathscr{A}, \mathscr{B} \in \operatorname{SVN}(\Omega)$, and we have the following relation, union, and intersection operations.

For Type-1,

(1) $\mathscr{A} \subseteq \mathscr{B} \Leftrightarrow \mathscr{T}_{\mathscr{A}} \leq \mathscr{T}_{\mathscr{B}}, \mathscr{I}_{\mathscr{B}} \leq \mathscr{I}_{\mathscr{A}}, \mathscr{F}_{\mathscr{A}} \leq \mathscr{F}_{\mathscr{B}} \forall x \in \Omega$

(2) $\mathscr{A} \cap \mathscr{B}=\left\{\left\langle x, \mathscr{T}_{\mathscr{A}} \wedge \mathscr{T}_{\mathscr{B}}, \mathscr{I}_{\mathscr{A}} \vee \mathscr{I}_{\mathscr{B}}, \mathscr{F}_{\mathscr{A}} \vee \mathscr{F}_{\mathscr{B}}\right\rangle\right\}$

(3) $\mathscr{A} \cup \mathscr{B}=\left\{\left\langle x, \mathscr{T}_{\mathscr{A}} \vee \mathscr{T}_{\mathscr{B}}, \mathscr{I}_{\mathscr{A}} \wedge \mathscr{I}_{\mathscr{B}}, \mathscr{F}_{\mathscr{A}} \wedge \mathscr{F}_{\mathscr{B}}\right\rangle\right\}$

For Type-2,

(1) $\mathscr{A} \sqsubseteq \mathscr{B} \Leftrightarrow \mathscr{T}_{\mathscr{A}} \leq \mathscr{T}_{\mathscr{B}}, \mathscr{I}_{\mathscr{B}} \leq \mathscr{I}_{\mathscr{A}}, \mathscr{F}_{\mathscr{A}} \leq \mathscr{F}_{\mathscr{B}} \forall x \in \Omega$

(2) $\mathscr{A} \sqcap \mathscr{B}=\left\{\left\langle x, \mathscr{T}_{\mathscr{A}} \wedge \mathscr{T}_{\mathscr{B}}, \mathscr{I}_{\mathscr{A}} \wedge \mathscr{I}_{\mathscr{B}}, \mathscr{F}_{\mathscr{A}} \vee \mathscr{F}_{\mathscr{B}}\right\rangle\right\}$

(3) $\mathscr{A} \sqcup \mathscr{B}=\left\{\left\langle x, \mathscr{T}_{\mathscr{A}} \vee \mathscr{T}_{\mathscr{B}}, \mathscr{I}_{\mathscr{A}} \vee \mathscr{I}_{\mathscr{B}}, \mathscr{F}_{\mathscr{A}} \wedge \mathscr{F}_{\mathscr{B}}\right\rangle\right\}$

Definition 5 (Cf. $[46,47])$. Let $(\Omega, \widetilde{\Gamma})$ be a 1-SVN $\beta$ CAS with $\widetilde{\Gamma}=\left\{\widetilde{C}_{1}, \widetilde{C}_{2}, \ldots, \widetilde{C}_{m}\right\}$ for some $\beta=\langle a, b, c\rangle$. Then, for each $x \in \Omega$, define the Type-1 SVN $\beta$-neighborhood (the Type-2 SVN $\beta$-neighborhood) of $x$ as follows: 


$$
\begin{aligned}
\widetilde{1}_{1}^{\beta} & =\cap\left\{\widetilde{C}_{i} \in \widetilde{\Gamma}: \widetilde{C}_{i}(x) \geq \beta\right\} \\
& =\cap\left\{\widetilde{C}_{i} \in \widetilde{\Gamma}: \mathscr{T}_{\widetilde{C}_{i}} \geq a, \mathscr{I}_{\widetilde{C}_{i}} \leq b, \mathscr{F}_{\widetilde{C}_{i}} \leq c\right\}, \\
\widetilde{N}^{\beta} & =\sqcap\left\{\widetilde{C}_{i} \in \widetilde{\Gamma}: \widetilde{C}_{i}(x) \geqslant \beta\right\} \\
& =\sqcap\left\{\widetilde{C}_{i} \in \widetilde{\Gamma}: \mathscr{T}_{\widetilde{C}_{i}} \geq a, \mathscr{I}_{\widetilde{C}_{i}} \geq b, \mathscr{F}_{\widetilde{C}_{i}} \leq c\right\} .
\end{aligned}
$$

$$
\begin{aligned}
& \mathscr{L}_{1}^{1}(\mathscr{A})=\left\{\left\langle x, \wedge_{y \in \Omega}\left(\mathscr{F}_{1}{ }_{1} \widetilde{\mathcal{N}}_{x}^{\beta}(y) \vee \mathscr{T}_{\mathscr{A}}(y)\right), \wedge_{y \in \Omega}\left(\left(1-\mathscr{I}_{1} \widetilde{\mathcal{N}}_{x}^{\beta}(y)\right) \vee \mathscr{I}_{\mathscr{A}}(y)\right), \vee_{y \in \Omega}\left(\mathscr{T}_{1} \widetilde{\mathcal{N}}_{x}^{\beta}(y) \wedge \mathscr{F}_{\mathscr{A}}(y)\right)\right\rangle\right\}, \\
& \mathscr{U}_{1}^{1}(\mathscr{A})=\left\{\left\langle x, \vee_{y \in \Omega}\left(\mathscr{T}_{1} \widetilde{\mathcal{N}}_{x}^{\beta}(y) \wedge \mathscr{T}_{\mathscr{A}}(y)\right), \vee_{y \in \Omega}\left(\mathscr{I}_{1}{\widetilde{\mathcal{N}_{x}^{\beta}}}_{x}(y) \wedge \mathscr{I}_{\mathscr{A}}(y)\right), \wedge_{y \in \Omega}\left(\mathscr{F}_{1} \widetilde{\mathcal{N}}_{x}^{\beta}(y) \vee \mathscr{F}_{\mathscr{A}}(y)\right)\right\rangle\right\}, \\
& \mathscr{L}_{1}^{2}(\mathscr{A})=\left\{\left\langle x, \wedge_{y \in \Omega}\left(\mathscr{F}_{2}{ }_{2} \widetilde{N}_{x}^{\beta}(y) \vee \mathscr{T}_{\mathscr{A}}(y)\right), \wedge_{y \in \Omega}\left(\left(1-\mathscr{I}_{2} \widetilde{\mathcal{N}}_{x}^{\beta}(y)\right) \vee \mathscr{I}_{\mathscr{A}}(y)\right), \vee_{y \in \Omega}\left(\mathscr{T}_{2} \widetilde{\mathcal{N}}_{x}^{\beta}(y) \wedge \mathscr{F}_{\mathscr{A}}(y)\right)\right\rangle\right\}, \\
& \mathscr{U}_{1}^{2}(\mathscr{A})=\left\{\left\langle x, \vee_{y \in \Omega}\left(\mathscr{T}_{2} \widetilde{\mathcal{N}}_{x}^{\beta}(y) \wedge \mathscr{T}_{\mathscr{A}}(y)\right), \vee_{y \in \Omega}\left(\mathscr{I}_{{ }_{2} \widetilde{N}_{x}^{\beta}}(y) \wedge \mathscr{F}_{\mathscr{A}}(y)\right), \wedge_{y \in \Omega}\left(\mathscr{F}_{2} \tilde{N}_{x}^{\beta}(y) \vee \mathscr{F}_{\mathscr{A}}(y)\right)\right\rangle\right\} .
\end{aligned}
$$

If $\mathscr{L}_{1}^{1}(\mathscr{A}) \quad$ (resp., $\left.\mathscr{L}_{1}^{2}(\mathscr{A})\right) \neq \mathscr{U}_{1}^{1}(\mathscr{A}) \quad$ (resp., $\mathscr{U}_{1}^{2}(\mathscr{A})$ ), then $\mathscr{A}$ is called the first type of Type-1 SVN $\beta$-covering rough sets (resp., the first type of Type-2 SVN $\beta$-covering rough sets) (briefly, 1-1-SVN $\beta$ CRSs (resp., 1-2SVN $\beta$ CRSs)).

\section{Type-1 SVN Complementary $\beta$-Neighborhood and Three New Kinds of Type-1 SVN $\beta$-CRS}

We will propose the concept of a type-1 SVN complementary $\beta$-neighborhood and three new kinds of Type-1 $\operatorname{SVN} \beta$-CRS and introduce several definitions, propositions, and examples as indicated below.

Definition 7. Let $(\Omega, \widetilde{\Gamma})$ be a 1-SVN $\beta$ CAS with $\widetilde{\Gamma}=\left\{\widetilde{C}_{1}, \widetilde{C}_{2}, \ldots, \widetilde{C}_{m}\right\}$, for some $\beta=\langle a, b, c\rangle$. Then, for each $x \in \Omega$, define the type- 1 SVN complementary $\beta$-neighborhood of $x$ as follows:

$$
{ }_{1} \widetilde{M}_{x}^{\beta}(y)={ }_{1} \widetilde{\mathcal{N}}_{y}^{\beta}(x), \quad \forall y \in \Omega .
$$

Example 1. Let $(\Omega, \widetilde{\Gamma})$ be a 1-SVN $\beta$ CAS, $\Omega=\left\{x_{1}, x_{2}, x_{3}, x_{4}, x_{5}\right\}$ and $\widetilde{\Gamma}=\left\{\widetilde{C}_{1}, \widetilde{C}_{2}, \widetilde{C}_{3}, \widetilde{C}_{4}\right\}$, where $\beta=$ $\langle 0.5,0.3,0.8\rangle$ are summarized in Table 1 .

In Table $1,{ }_{1} \widetilde{\mathscr{N}}_{x_{1}}^{\beta}=\widetilde{C}_{1} \cap \widetilde{C}_{2}, \widetilde{1}_{1}^{\mathcal{N}_{x}}=\widetilde{C}_{1} \cap \widetilde{C}_{2} \cap \widetilde{C}_{4}$, $\widetilde{ }_{1} \widetilde{N}_{x_{3}}^{\beta}=\widetilde{C}_{3} \cap \widetilde{C}_{4}, \widetilde{N}_{x_{4}}^{\beta}=\widetilde{C}_{1} \cap \widetilde{C}_{4}$, and ${\widetilde{{ }_{1}}}_{x_{5}}^{\beta}=\widetilde{C}_{2} \cap \widetilde{C}_{3} \cap \widetilde{C}_{4}$.

Table 2 contains the results of type-1 SVN $\beta$-neighborhood.

Thus, we can obtain the values of type-1 SVN complementary $\beta$-neighborhood as in Table 3 .

Hence, we can merge ${ }_{1} \widetilde{\mathcal{N}}_{x}^{\beta}$ and ${ }_{1} \dot{\mathscr{M}}_{x}^{\beta}$ and compute ${ }_{1} \widetilde{\mathcal{N}}_{x}^{\beta} \cap{ }_{1} \widetilde{\mathscr{M}}_{x}^{\beta}$ as Table 4.
Also, we can compute ${ }_{1} \widetilde{\mathcal{N}}_{x}^{\beta} \cup{ }_{1} \widetilde{M}_{x}^{\beta}$ as set in Table 5.

Proposition 1. Let $(\Omega, \widetilde{\Gamma})$ be a 1-SVN $\beta C A S$, for some $\beta=$ $\langle a, b, c\rangle$ and for each $x, y, z \in \Omega$. Then, the following statements hold:
(1) ${ }_{1} \widetilde{M}_{x}^{\beta}(x) \geq \beta$
(2) If ${ }_{1} \mathscr{M}_{x}^{\beta}(y) \geq \beta$ and ${ }_{1} \widetilde{M}_{y}^{\beta}(z) \geq \beta$, then ${ }_{1} \widetilde{M}_{x}^{\beta}(z) \geq \beta$
(3) $0<\beta_{1} \leq \beta_{2} \leq \beta$, then ${ }_{1} \widetilde{\mathscr{M}}_{x}^{\beta_{1}} \subseteq{ }_{1} \mathscr{M}_{x}^{\beta_{2}}$

Proof

(1) It follows directly from Definitions 5 and 7.

(2) Since ${ }_{1} \widetilde{M}_{x}^{\beta}(y) \geq \beta$, then ${ }_{1} \widetilde{N}_{y}^{\beta}(x) \geq \beta$. If $\widetilde{C}_{i}(x) \geq \beta$, then $\widetilde{C}_{i}(y) \geq \beta$, and since $\widetilde{\mathscr{M}}_{y}^{\beta}(z) \geq \beta$, then ${ }_{1} \widetilde{\mathcal{N}}_{z}^{\beta}(y) \geq \beta$. If $\widetilde{C}_{i}(y) \geq \beta$, then $\widetilde{C}_{i}(z) \geq \beta$. Therefore, ${ }_{1} \widetilde{M}_{x}^{\beta}(z) \geq \beta$.

(3) For each $x \in \Omega, 0<\beta_{1} \leq \beta_{2} \leq \beta$, then $\cap\left\{\widetilde{C}_{i} \in\right.$ $\left.\widetilde{\Gamma}: \widetilde{C}_{i}(x) \geq \beta_{1}\right\} \supseteq \cap\left\{\widetilde{C}_{i} \in \widetilde{\Gamma}: \widetilde{C}_{i}(x) \geq \beta_{2}\right\}$. Thus, by Definition 7, we have ${ }_{1} \widetilde{\mathscr{M}}_{x}^{\beta_{1}} \subseteq_{1} \widetilde{\mathscr{M}}_{x}^{\beta_{2}}$.

Proposition 2. Let $(\Omega, \widetilde{\Gamma})$ be a 1-SVN $\beta C A S$, for some $\beta=\langle a, b, c\rangle$. For each $x, y \in \Omega$,

$$
{ }_{1} \widetilde{M}_{x}^{\beta}(y) \geq \beta \Leftrightarrow{ }_{1} \widetilde{M}_{y}^{\beta} \subseteq_{1} \widetilde{M}_{x}^{\beta} \text {. }
$$

Proof. Let ${ }_{1} \widetilde{\mathscr{M}}_{x}^{\beta}(y) \geq \beta, \mathscr{T}_{{ }_{1} \tilde{M}_{x}^{\beta}}(y)=\mathscr{T} \cap \widetilde{C}_{i}(y)=\wedge \mathscr{T}_{\widetilde{C}_{i}}$ $(y) \geq a, \mathscr{I}_{1 \tilde{\mathbb{M}}_{x}^{\beta}}(y)=\mathscr{I} \cap \widetilde{C}_{i}(y)=\vee \mathscr{I}_{\widetilde{C}_{i}}(y) \leq b$, and $\mathscr{F}_{1} \tilde{\mathscr{M}}_{x}^{\beta}$ $(y)=\mathscr{F} \cap \widetilde{C}_{i}(y)=\vee \mathscr{F}_{\widetilde{C}_{i}}(y) \leq c$. Then, $\left\{\widetilde{C}_{i} \in \widetilde{\Gamma}: \mathscr{T}_{\widetilde{C}_{i}}(x) \geq a\right.$, $\left.\mathscr{I}_{\widetilde{C}_{i}}(x) \leq b, \quad \mathscr{F}_{\widetilde{C}_{i}}(x) \leq c\right\} \subseteq\left\{\widetilde{C}_{i} \in \widetilde{\Gamma}: \mathscr{T}_{\widetilde{C}_{i}}(y) \geq a, \mathscr{I}_{\widetilde{C}_{i}}(y) \leq\right.$ 
TABle 1: $(\Omega, \widetilde{\Gamma})$.

\begin{tabular}{ccccc}
\hline & $\widetilde{C}_{1}$ & $\widetilde{C}_{2}$ & $\widetilde{C}_{3}$ & $\widetilde{C}_{4}$ \\
\hline$x_{1}$ & $\langle 0.7,0.2,0.5\rangle$ & $\langle 0.6,0.2,0.4\rangle$ & $\langle 0.4,0.1,0.5\rangle$ & $\langle 0.1,0.5,0.6\rangle$ \\
$x_{2}$ & $\langle 0.5,0.3,0.2\rangle$ & $\langle 0.5,0.2,0.8\rangle$ & $\langle 0.4,0.5,0.4\rangle$ & $\langle 0.6,0.1,0.7\rangle$ \\
$x_{3}$ & $\langle 0.4,0.5,0.2\rangle$ & $\langle 0.2,0.3,0.6\rangle$ & $\langle 0.5,0.2,0.4\rangle$ & $\langle 0.6,0.3,0.4\rangle$ \\
$x_{4}$ & $\langle 0.6,0.1,0.7\rangle$ & $\langle 0.4,0.5,0.7\rangle$ & $\langle 0.3,0.6,0.5\rangle$ & $\langle 0.5,0.3,0.2\rangle$ \\
$x_{5}$ & $\langle 0.3,0.2,0.6\rangle$ & $\langle 0.7,0.3,0.5\rangle$ & $\langle 0.6,0.3,0.5\rangle$ & $\langle 0.8,0.1,0.2\rangle$ \\
\hline
\end{tabular}

TABLE $2: \widetilde{\mathcal{N}}_{x_{i}}^{\beta}, \forall i=1,2, \ldots, 5$.

\begin{tabular}{|c|c|c|c|c|c|}
\hline & $x_{1}$ & $x_{2}$ & $x_{3}$ & $x_{4}$ & $x_{5}$ \\
\hline${\widetilde{{ }_{1} \mathcal{N}_{x_{1}}^{\beta}}}_{\beta}^{\beta}$ & $\langle 0.6,0.2,0.5\rangle$ & $\langle 0.5,0.3,0.8\rangle$ & $\langle 0.2,0.5,0.6\rangle$ & $\langle 0.4,0.5,0.7\rangle$ & $\langle 0.3,0.3,0.6\rangle$ \\
\hline$\widetilde{N}_{{ }_{1}}^{\beta}$ & $\langle 0.1,0.5,0.6\rangle$ & $\langle 0.5,0.3,0.8\rangle$ & $\langle 0.2,0.5,0.6\rangle$ & $\langle 0.4,0.5,0.7\rangle$ & $\langle 0.3,0.3,0.6\rangle$ \\
\hline$\widetilde{N}_{x_{3}}^{\beta^{2}}$ & $\langle 0.1,0.5,0.6\rangle$ & $\langle 0.4,0.5,0.7\rangle$ & $\langle 0.5,0.3,0.4\rangle$ & $\langle 0.3,0.6,0.5\rangle$ & $\langle 0.6,0.3,0.5\rangle$ \\
\hline$\widetilde{1}_{1}^{\beta}{ }_{x_{4}}^{\beta}$ & $\langle 0.1,0.5,0.6\rangle$ & $\langle 0.5,0.3,0.7\rangle$ & $\langle 0.4,0.5,0.4\rangle$ & $\langle 0.5,0.3,0.7\rangle$ & $\langle 0.3,0.2,0.6\rangle$ \\
\hline$\widetilde{1}_{{ }_{1}}^{\beta}$ & $\langle 0.1,0.5,0.6\rangle$ & $\langle 0.4,0.5,0.8\rangle$ & $\langle 0.2,0.3,0.6\rangle$ & $\langle 0.3,0.6,0.7\rangle$ & $\langle 0.6,0.3,0.5\rangle$ \\
\hline
\end{tabular}

TABle 3: $\widetilde{\mathscr{M}}_{x_{i}}^{\beta}, \forall i=1,2, \ldots, 5$.

\begin{tabular}{cccccc}
\hline & $x_{1}$ & $x_{2}$ & $x_{3}$ & $x_{4}$ & $x_{5}$ \\
\hline$\widetilde{\mathscr{M}}_{x_{1}}^{\beta}$ & $\langle 0.5,0.8,0.6\rangle$ & $\langle 0.8,0.7,0.5\rangle$ & $\langle 0.6,0.5,0.2\rangle$ & $\langle 0.7,0.5,0.4\rangle$ & $\langle 0.6,0.7,0.3\rangle$ \\
$\widetilde{M}_{1}^{\beta} \tilde{M}_{x_{2}}$ & $\langle 0.6,0.5,0.1\rangle$ & $\langle 0.8,0.7,0.5\rangle$ & $\langle 0.6,0.5,0.2\rangle$ & $\langle 0.7,0.5,0.4\rangle$ & $\langle 0.6,0.7,0.3\rangle$ \\
${ }_{1} \mathscr{M}_{x_{3}}^{\beta}$ & $\langle 0.6,0.5,0.1\rangle$ & $\langle 0.7,0.5,0.4\rangle$ & $\langle 0.4,0.7,0.5\rangle$ & $\langle 0.5,0.4,0.3\rangle$ & $\langle 0.5,0.7,0.6\rangle$ \\
$\tilde{}_{1} \mathscr{M}_{x_{4}}^{\beta}$ & $\langle 0.6,0.5,0.1\rangle$ & $\langle 0.7,0.7,0.5\rangle$ & $\langle 0.4,0.5,0.4\rangle$ & $\langle 0.7,0.7,0.5\rangle$ & $\langle 0.6,0.8,0.3\rangle$ \\
$\tilde{}_{1}^{\beta} \mathscr{M}_{x_{5}}^{\beta}$ & $\langle 0.6,0.5,0.1\rangle$ & $\langle 0.8,0.5,0.4\rangle$ & $\langle 0.6,0.7,0.2\rangle$ & $\langle 0.7,0.4,0.3\rangle$ & $\langle 0.5,0.7,0.6\rangle$ \\
\hline
\end{tabular}

TABLE 4: $\widetilde{\mathscr{N}}_{x_{i}}^{\beta} \cap \widetilde{\mathscr{M}}_{x_{i}}^{\beta}, \forall i=1,2, \ldots, 5$.

\begin{tabular}{|c|c|c|c|c|c|}
\hline & $x_{1}$ & $x_{2}$ & $x_{3}$ & $x_{4}$ & $x_{5}$ \\
\hline$\widetilde{\mathcal{N}}_{x_{1}}^{\beta} \cap \widetilde{\mathscr{M}}_{x_{1}}^{\beta}$ & $\langle 0.5,0.8,0.6\rangle$ & $\langle 0.5,0.7,0.8\rangle$ & $\langle 0.2,0.5,0.6\rangle$ & $\langle 0.4,0.5,0.7\rangle$ & $\langle 0.3,0.7,0.6\rangle$ \\
\hline$\widetilde{N}_{{ }_{1}}^{\beta}{ }^{1} \cap \widetilde{M}_{x_{1}}^{\beta}$ & $\langle 0.1,0.5,0.6\rangle$ & $\langle 0.5,0.7,0.8\rangle$ & $\langle 0.2,0.5,0.6\rangle$ & $\langle 0.4,0.5,0.7\rangle$ & $\langle 0.3,0.7,0.6\rangle$ \\
\hline$\widetilde{F}_{1}^{\beta}{ }_{x_{3}}^{\beta} \cap \widetilde{M}_{x_{3}}^{\beta}$ & $\langle 0.1,0.5,0.6\rangle$ & $\langle 0.4,0.5,0.7\rangle$ & $\langle 0.4,0.7,0.5\rangle$ & $\langle 0.3,0.6,0.5\rangle$ & $\langle 0.5,0.7,0.6\rangle$ \\
\hline$\widetilde{T}_{1}^{\beta} \cap \widetilde{N}_{x_{4}}^{\beta} \widetilde{M}_{x_{4}}^{\beta}$ & $\langle 0.1,0.5,0.6\rangle$ & $\langle 0.5,0.7,0.7\rangle$ & $\langle 0.4,0.5,0.4\rangle$ & $\langle 0.5,0.7,0.7\rangle$ & $\langle 0.3,0.8,0.6\rangle$ \\
\hline$\widetilde{F}_{1}^{\beta}{ }_{x_{5}}^{\beta} \cap \widetilde{M}_{x_{5}}^{\beta}$ & $\langle 0.1,0.5,0.6\rangle$ & $\langle 0.4,0.5,0.8\rangle$ & $\langle 0.2,0.7,0.6\rangle$ & $\langle 0.3,0.6,0.7\rangle$ & $\langle 0.5,0.7,0.6\rangle$ \\
\hline
\end{tabular}

TABLE 5: $\widetilde{\mathscr{N}}_{x_{i}}^{\beta} \cup \widetilde{M}_{1}^{\beta}, \forall i=1,2, \ldots, 5$.

\begin{tabular}{|c|c|c|c|c|c|}
\hline & $x_{1}$ & $x_{2}$ & $x_{3}$ & $x_{4}$ & $x_{5}$ \\
\hline$\widetilde{\mathcal{N}}_{x_{1}}^{\beta} \cup \widetilde{\mathscr{M}}_{x_{1}}^{\beta}$ & $\langle 0.6,0.2,0.5\rangle$ & $\langle 0.8,0.3,0.5\rangle$ & $\langle 0.6,0.5,0.2\rangle$ & $\langle 0.7,0.5,0.4\rangle$ & $\langle 0.6,0.3,0.3\rangle$ \\
\hline$\widetilde{1}_{1}^{\beta} \mathcal{N}_{x_{2}}^{\beta} \cup \widetilde{M}_{1}^{\beta}$ & $\langle 0.6,0.5,0.1\rangle$ & $\langle 0.8,0.3,0.5\rangle$ & $\langle 0.6,0.5,0.2\rangle$ & $\langle 0.7,0.5,0.4\rangle$ & $\langle 0.6,0.3,0.3\rangle$ \\
\hline$\widetilde{\mathcal{N}}_{x_{3}}^{\beta} \cup \widetilde{\mathscr{M}}_{x_{3}}^{\beta}$ & $\langle 0.6,0.5,0.1\rangle$ & $\langle 0.7,0.5,0.4\rangle$ & $\langle 0.5,0.3,0.4\rangle$ & $\langle 0.5,0.4,0.3\rangle$ & $\langle 0.6,0.3,0.5\rangle$ \\
\hline$\widetilde{\mathcal{N}}_{x_{4}}^{\beta} \cup \widetilde{M}_{1}^{\beta}{ }_{x_{4}}^{\beta}$ & $\langle 0.6,0.5,0.1\rangle$ & $\langle 0.7,0.3,0.5\rangle$ & $\langle 0.4,0.5,0.4\rangle$ & $\langle 0.7,0.3,0.5\rangle$ & $\langle 0.6,0.2,0.3\rangle$ \\
\hline$\widetilde{\mathcal{N}}_{x_{5}}^{\beta} \cup \widetilde{M}_{x_{5}}^{\beta}$ & $\langle 0.6,0.5,0.1\rangle$ & $\langle 0.8,0.5,0.4\rangle$ & $\langle 0.6,0.3,0.2\rangle$ & $\langle 0.7,0.4,0.3\rangle$ & $\langle 0.6,0.3,0.5\rangle$ \\
\hline
\end{tabular}


$\left.b, \mathscr{F}_{\widetilde{C}_{i}}(y) \leq c\right\}$. Thus, $\widetilde{M}_{1}^{\beta} \subseteq_{1} \widetilde{\mathscr{M}}_{x}^{\beta}$. On the contrary, let ${ }_{1} \widetilde{M}_{y}^{\beta} \subseteq_{1} \widetilde{\mathscr{M}}_{x}^{\beta}$. Then, $\mathscr{T}_{{ }_{1}} \widetilde{\mathbb{M}}_{x}^{\beta}(y) \geq \mathscr{T}_{1} \widetilde{\mathbb{M}}_{y}^{\beta}(y) \geq a, \mathscr{I}_{1} \widetilde{\mathbb{M}}_{x}^{\beta}(y) \leq$ $\mathscr{I}{ }_{2 \beta} \widetilde{\mathbb{M}}^{\beta}{ }^{\beta}(y) \leq b$, and $\mathscr{F}{ }_{1} \widetilde{\mathbb{M}}_{x}^{\beta}(y) \leq \mathscr{F}_{1}{ }_{1} \widetilde{\mathbb{M}}_{y}^{\beta}(y) \leq c$. Hence, ${ }_{1}{ }^{1} \mathscr{M}_{x}^{\beta}(y)$

Now, we present the three new types of 1-1-SVN $\beta$ CRSs based on Definitions 5 and 7 as indicated below.
Definition 8. Consider $(\Omega, \widetilde{\Gamma})$ is a 1 -SVN $\beta$ CAS with $\widetilde{\Gamma}=\left\{\widetilde{C}_{1}, \widetilde{C}_{2}, \ldots, \widetilde{C}_{m}\right\}$, for some $\beta=\langle a, b, c\rangle$. For each $x \in \Omega$ and $\mathscr{A} \in \operatorname{SVN}(\Omega)$, then we have the following paradigms:

Paradigm 1: the second type of Type-1 SVN lower approximation (2-1-SVNLA) $\mathscr{L}_{2}^{1}(\mathscr{A})$ and the second type of Type-1 SVN upper approximation (2-1SVNUA) $\mathscr{U}_{2}^{1}(\mathscr{A})$ are as follows:

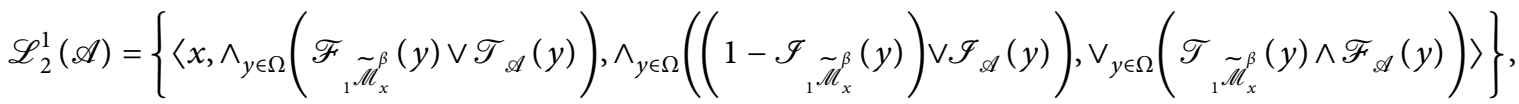

$$
\begin{aligned}
& \mathscr{U}_{2}^{1}(\mathscr{A})=\left\{\left\langle x, \vee_{y \in \Omega}\left(\mathscr{T}_{1} \tilde{\mathbb{M}}_{x}^{\beta}(y) \wedge \mathscr{T}_{\mathscr{A}}(y)\right), \vee_{y \in \Omega}\left(\mathscr{I}_{1} \tilde{\mathscr{M}}_{x}^{\beta}(y) \wedge \mathscr{I}_{\mathscr{A}}(y)\right), \wedge_{y \in \Omega}\left(\mathscr{F}_{1} \tilde{\mathbb{M}}_{x}^{\beta}(y) \vee \mathscr{F}_{\mathscr{A}}(y)\right)\right\rangle\right\} .
\end{aligned}
$$

If $\mathscr{L}_{2}^{1}(\mathscr{A}) \neq \mathscr{U}_{2}^{1}(\mathscr{A})$, then $\mathscr{A}$ is called the second type of Type-1 SVN $\beta$-covering rough sets (briefly, 2-1SVN $\beta$ CRSs).
Paradigm 2: the third type of Type-1 SVN lower approximation (3-1-SVNLA) $\mathscr{L}_{3}^{1}(\mathscr{A})$ and the third type of Type-1 SVN upper approximation (3-1-SVNUA) $\mathcal{U}_{3}^{1}(\mathscr{A})$ are introduced as follows:

$$
\begin{aligned}
& \mathscr{L}_{3}^{1}(\mathscr{A})=\left\{\left\langle x, \wedge_{y \in \Omega}\left(\mathscr{F}_{1} \tilde{\mathcal{N}}_{x}^{\beta} \cap_{1} \tilde{\mathcal{M}}_{x}^{\beta}(y) \vee \mathscr{T}_{\mathscr{A}}(y)\right), \wedge_{y \in \Omega}\left(\left(1-\mathscr{F}_{{ }_{1} \tilde{\mathcal{N}}_{x}^{\beta} \cap_{1} \tilde{\mathcal{H}}_{x}^{\beta}}(y)\right) \vee \mathscr{F}_{\mathscr{A}}(y)\right), \vee_{y \in \Omega}\left(\mathscr{T}_{1} \tilde{\mathcal{N}}_{x}^{\beta} \cap_{1} \tilde{\mathscr{M}}_{x}^{\beta}(y) \wedge \mathscr{F}_{\mathscr{A}}(y)\right)\right\rangle\right\},
\end{aligned}
$$

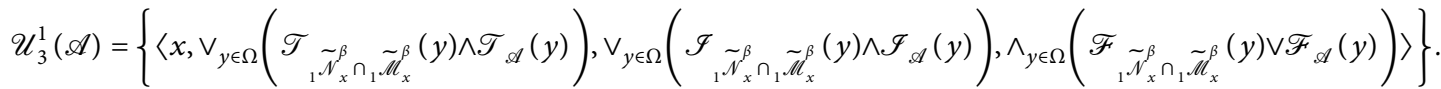

If $\mathscr{L}_{3}^{1}(\mathscr{A}) \neq \mathscr{U}_{3}^{1}(\mathscr{A})$, then $\mathscr{A}$ is called the third type of Type-1 SVN $\beta$-covering rough sets (briefly, 3-1SVN $\beta$ CRSs).
Paradigm 3: the fourth type of Type-1 SVN lower approximation (4-1-SVNLA) $\mathscr{L}_{4}^{1}(\mathscr{A})$ and the fourth type of Type-1 SVN upper approximation (4-1SVNUA) $\mathscr{U}_{4}^{1}(\mathscr{A})$ are proposed as follows:

$$
\begin{aligned}
& \mathscr{L}_{4}^{1}(\mathscr{A})=\left\{\left\langle x, \wedge_{y \in \Omega}\left(\mathscr{F}_{1} \tilde{\mathcal{N}}_{x}^{\beta} \cup_{1} \tilde{\mathscr{M}}_{x}^{\beta}(y) \vee \mathscr{T}_{\mathscr{A}}(y)\right), \wedge_{y \in \Omega}\left(\left(1-\mathscr{I}_{{ }_{1}} \tilde{\mathcal{N}}_{x}^{\beta} \cup_{1} \tilde{\mathcal{M}}_{x}^{\beta}(y)\right) \vee \mathscr{F}_{\mathscr{A}}(y)\right), \vee_{y \in \Omega}\left(\mathscr{T}_{{ }_{1} \tilde{\mathcal{N}}_{x}^{\beta} \cup_{1} \tilde{M}_{x}^{\beta}}(y) \wedge \mathscr{F}_{\mathscr{A}}(y)\right)\right\rangle\right\},
\end{aligned}
$$

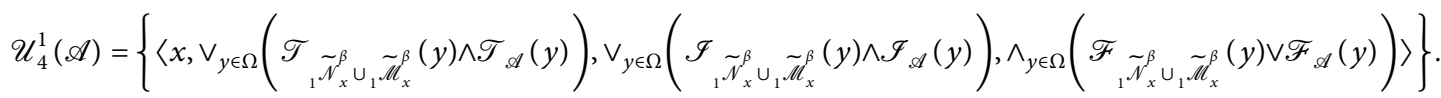

If $\mathscr{L}_{4}^{1}(\mathscr{A}) \neq \mathscr{U}_{4}^{1}(\mathscr{A})$, then $\mathscr{A}$ is called the fourth type of Type-1 SVN $\beta$-covering rough sets (briefly, 4 -1-SVN $\beta$ CRSs).
Example 2. Consider Example 1 if $\beta=\langle 0.5,0.3,0.8\rangle$ and $\mathscr{A}=\left((0.5,0.3,0.6) / x_{1}\right)+\left((0.4,0.5, \quad 0.1) / x_{2}\right)+((0.3,0.2$, 
$\left.0.6) / x_{3}\right)+\left((0.5,0.3,0.4) / x_{4}\right)+\left((0.7,0.2,0.3) / x_{5}\right)$; then, we have the following results:

$$
\begin{aligned}
& \mathscr{L}_{1}^{1}(\mathscr{A})=\left\{\left\langle x_{1}, 0.6,0.5,0.5\right\rangle,\left\langle x_{2}, 0.6,0.5,0.4\right\rangle,\left\langle x_{3}, 0.4,0.4,0.5\right\rangle,\left\langle x_{4}, 0.4,0.5,0.4\right\rangle,\left\langle x_{5}, 0.6,0.4,0.3\right\rangle\right\}, \\
& \mathscr{U}_{1}^{1}(\mathscr{A})=\left\{\left\langle x_{1}, 0.6,0.3,0.5\right\rangle,\left\langle x_{2}, 0.4,0.3,0.6\right\rangle,\left\langle x_{3}, 0.6,0.5,0.5\right\rangle,\left\langle x_{4}, 0.5,0.3,0.6\right\rangle,\left\langle x_{5}, 0.6,0.5,0.5\right\rangle\right\}, \\
& \mathscr{L}_{2}^{1}(\mathscr{A})=\left\{\left\langle x_{1}, 0.3,0.3,0.6\right\rangle,\left\langle x_{2}, 0.3,0.3,0.6\right\rangle,\left\langle x_{3}, 0.4,0.3,0.5\right\rangle,\left\langle x_{4}, 0.4,0.2,0.4\right\rangle,\left\langle x_{5}, 0.3,0.3,0.6\right\rangle\right\}, \\
& \mathscr{U}_{2}^{1}(\mathscr{A})=\left\{\left\langle x_{1}, 0.6,0.5,0.3\right\rangle,\left\langle x_{2}, 0.6,0.5,0.3\right\rangle,\left\langle x_{3}, 0.6,0.5,0.4\right\rangle,\left\langle x_{4}, 0.6,0.5,0.3\right\rangle,\left\langle x_{5}, 0.6,0.5,0.4\right\rangle\right\}, \\
& \mathscr{L}_{3}^{1}(\mathscr{A})=\left\{\left\langle x_{1}, 0.6,0.3,0.5\right\rangle,\left\langle x_{2}, 0.6,0.3,0.4\right\rangle,\left\langle x_{3}, 0.5,0.3,0.4\right\rangle,\left\langle x_{4}, 0.4,0.2,0.3\right\rangle,\left\langle x_{5}, 0.6,0.3,0.3\right\rangle\right\}, \\
& \mathscr{U}_{3}^{1}(\mathscr{A})=\left\{\left\langle x_{1}, 0.5,0.5,0.6\right\rangle,\left\langle x_{2}, 0.4,0.5,0.6\right\rangle,\left\langle x_{3}, 0.5,0.5,0.5\right\rangle,\left\langle x_{4}, 0.5,0.5,0.6\right\rangle,\left\langle x_{5}, 0.5,0.5,0.6\right\rangle\right\}, \\
& \mathscr{L}_{4}^{1}(\mathscr{A})=\left\{\left\langle x_{1}, 0.3,0.5,0.6\right\rangle,\left\langle x_{2}, 0.3,0.5,0.6\right\rangle,\left\langle x_{3}, 0.4,0.5,0.5\right\rangle,\left\langle x_{4}, 0.4,0.5,0.5\right\rangle,\left\langle x_{5}, 0.3,0.5,0.6\right\rangle\right\}, \\
& \mathscr{U}_{4}^{1}(\mathscr{A})=\left\{\left\langle x_{1}, 0.6,0.3,0.3\right\rangle,\left\langle x_{2}, 0.6,0.3,0.3\right\rangle,\left\langle x_{3}, 0.6,0.5,0.4\right\rangle,\left\langle x_{4}, 0.6,0.3,0.3\right\rangle,\left\langle x_{5}, 0.6,0.5,0.4\right\rangle\right\} .
\end{aligned}
$$

Next, we will present Proposition 3 for the 2-1SVN $\beta$ CRS model; also, it satisfies in case of the 3-1SVN $\beta$ CRS and the 4-1-SVN $\beta$ CRS models.

Proposition 3. Let $(\Omega, \widetilde{\Gamma})$ be a 1-SVN $\beta C A S$, for some $\beta=\langle a, b, c\rangle$. For each $x, y, z \in \Omega$ and $\mathscr{A}, \mathscr{B} \in \operatorname{SVN}(\Omega)$. Then, the following statements hold:

(1) $(S V N L 1) \mathscr{L}_{2}^{1}\left(\mathscr{A}^{c}\right)=\left(\mathscr{U}_{2}^{1}(\mathscr{A})\right)^{c}$. $(S V N U 1) \mathscr{U}_{2}^{1}\left(\mathscr{A}^{c}\right)=\left(\mathscr{L}_{2}^{1}(\mathscr{A})\right)^{c}$.

(2) If $\mathscr{A} \subseteq \mathscr{B}$, then $(S V N L 2) \mathscr{L}_{2}^{1}(\mathscr{A}) \subseteq \mathscr{L}_{2}^{1}(\mathscr{B})$.
(SVNU2) $\mathcal{U}_{2}^{1}(\mathscr{A}) \subseteq \mathcal{U}_{2}^{1}(\mathscr{B})$.

(3) $(S V N L 3) \mathscr{L}_{2}^{1}(\mathscr{A} \cap \mathscr{B})=\mathscr{L}_{2}^{1}(\mathscr{A}) \cap \mathscr{L}_{2}^{1}(\mathscr{B})$. $(S V N U 3) \mathcal{U}_{2}^{1}(\mathscr{A} \cap \mathscr{B}) \subseteq \mathcal{U}_{2}^{1}(\mathscr{A}) \cap \mathcal{U}_{2}^{1}(\mathscr{B})$.

(4) $(S V N L 4) \mathscr{L}_{2}^{1}(\mathscr{A} \cup \mathscr{B}) \supseteq \mathscr{L}_{2}^{1}(\mathscr{A}) \cup \mathscr{L}_{2}^{1}(\mathscr{B})$. $(\operatorname{SVNU} 4) \mathcal{U}_{2}^{1}(\mathscr{A} \cap \mathscr{B})=\mathcal{U}_{2}^{1}(\mathscr{A}) \cap \mathcal{U}_{2}^{1}(\mathscr{B})$.

Proof. We shall only prove (SVNL1), (SVNL2), (SVNL3), and (SVNL4).

(SVNL1):

$$
\begin{aligned}
\mathscr{L}_{2}^{1}\left(\mathscr{A}^{c}\right) & =\left\{\left\langle x, \wedge_{y \in \Omega}\left(\mathscr{F}_{1} \underset{{ }_{1} \tilde{M}_{x}^{\beta}}{ }(y) \vee \mathscr{T}_{\mathscr{A}^{c}}(y)\right), \wedge_{y \in \Omega}\left(\left(1-\mathscr{I}_{1} \tilde{\mathscr{M}}_{x}^{\beta}(y)\right) \vee \mathscr{I}_{\mathscr{A}^{c}}(y)\right), \vee_{y \in \Omega}\left(\mathscr{T}_{1} \tilde{\mathscr{M}}_{x}^{\beta}(y) \wedge \mathscr{F}_{\mathscr{A}^{c}}(y)\right)\right\rangle\right\} \\
& =\left\{\left\langle x, \wedge_{y \in \Omega}\left(\mathscr{F}_{{ }_{1}} \underset{\tilde{M}_{x}^{\beta}}{ }(y) \vee \mathscr{F}_{\mathscr{A}}(y)\right), \wedge_{y \in \Omega}\left(\left(1-\mathscr{I}_{{ }_{1} \tilde{\mathscr{M}}_{x}^{\beta}}(y)\right) \vee\left(1-\mathscr{I}_{\mathscr{A}}(y)\right)\right), \vee_{y \in \Omega}\left(\mathscr{T}_{1} \tilde{\mathscr{M}}_{x}^{\beta}(y) \wedge \mathscr{T}_{\mathscr{A}}(y)\right)\right\rangle\right\} \\
& =\left(\mathscr{U}_{2}^{1}(\mathscr{A})\right)^{c} .
\end{aligned}
$$

(SVNL2): let $\mathscr{A}, \mathscr{B} \in \operatorname{SVN}(\Omega)$ such that $\mathscr{A} \subseteq \mathscr{B}$ (i.e., $\mathscr{T}_{\mathscr{A}} \leq \mathscr{T}_{\mathscr{B}}, \mathscr{I}_{\mathscr{B}} \leq \mathscr{I}_{\mathscr{A}}$ and $\left.\mathscr{F}_{\mathscr{B}} \leq \mathscr{F}_{\mathscr{A}}\right)$ and $x \in \Omega$. Then, we get the following result:

$$
\begin{aligned}
& \mathscr{L}_{2}^{1}(\mathscr{A})(x)=\left\{\left\langle x, \wedge_{y \in \Omega}\left(\mathscr{F}_{{ }_{1}} \tilde{\mathscr{M}}_{x}^{\beta}(y) \vee \mathscr{T}_{\mathscr{A}}(y)\right), \wedge_{y \in \Omega}\left(\left(1-\mathscr{I}_{1} \tilde{\mathscr{M}}_{x}^{\beta}(y)\right) \vee \mathscr{I}_{\mathscr{A}}(y)\right), \vee_{y \in \Omega}\left(\mathscr{T}_{1} \tilde{\mathscr{M}}_{x}^{\beta}(y) \wedge \mathscr{F}_{\mathscr{A}}(y)\right)\right\rangle\right\}, \\
& \mathscr{T}_{\mathscr{L}_{2}^{1}(\mathscr{A})}=\wedge_{y \in \Omega}\left(\mathscr{F}_{1}{ }_{1} \tilde{\mathscr{M}}_{x}^{\beta}(y) \vee \mathscr{T}_{\mathscr{A}}(y)\right) \leq \wedge_{y \in \Omega}\left(\mathscr{F}_{1}{ }_{1} \tilde{\mathscr{M}}_{x}^{\beta}(y) \vee \mathscr{T}_{\mathscr{B}}(y)\right)=\mathscr{T}_{\mathscr{L}_{2}^{1}(\mathscr{B})}, \\
& \mathscr{I}_{\mathscr{L}_{2}^{1}(\mathscr{A})}=\wedge_{y \in \Omega}\left(\left(1-\mathscr{I}_{{ }_{1} \tilde{\mathscr{M}}_{x}^{\beta}}(y)\right) \vee \mathscr{I}_{\mathscr{A}}(y)\right) \geq \wedge_{y \in \Omega}\left(\left(1-\mathscr{I}_{1} \tilde{\mathscr{M}}_{x}^{\beta}(y)\right) \vee \mathscr{I}_{\mathscr{B}}(y)\right)=\mathscr{I}_{\mathscr{L}_{2}^{1}(\mathscr{B})}, \\
& \mathscr{F}_{\mathscr{L}_{2}^{1}(\mathscr{A})}=\vee_{y \in \Omega}\left(\mathscr{T}_{{ }_{1} \tilde{\mathscr{M}}_{x}^{\beta}}(y) \wedge \mathscr{F}_{\mathscr{A}}(y)\right) \geq \vee_{y \in \Omega}\left(\mathscr{T}_{1} \tilde{\mathscr{M}}_{x}^{\beta}(y) \wedge \mathscr{F}_{\mathscr{B}}(y)\right)=\mathscr{F}_{\mathscr{L}_{2}^{1}(\mathscr{B})} .
\end{aligned}
$$

Therefore, $\mathscr{L}_{2}^{1}(\mathscr{A}) \subseteq \mathscr{L}_{2}^{1}(\mathscr{B})$. 


$$
\begin{aligned}
& \mathscr{L}_{2}^{1}(\mathscr{A})(x)=\left\{\left\langle x, \wedge_{y \in \Omega}\left(\mathscr{F}_{1} \tilde{\mathscr{M}}_{x}^{\beta}(y) \vee \mathscr{T}_{\mathscr{A} \cap \mathscr{B}}(y)\right), \wedge_{y \in \Omega}\left(\left(1-\mathscr{I}_{1} \tilde{\mathbb{M}}_{x}^{\beta}(y)\right) \vee \mathscr{T}_{\mathscr{A} \cap \mathscr{B}}(y)\right), \vee_{y \in \Omega}\left(\mathscr{T}_{1} \tilde{M}_{x}^{\beta}(y) \wedge \mathscr{F}_{\mathscr{A} \cap \mathscr{B}}(y)\right)\right\rangle\right\} \\
& =\left\{\left\langle x, \wedge_{y \in \Omega}\left(\mathscr{F}_{1}{ }_{1}^{\beta}{ }_{x}(y) \vee\left(\mathscr{T}_{\mathscr{A}}(y) \wedge \mathscr{T}_{\mathscr{B}}(y)\right)\right), \wedge_{y \in \Omega}\left(\left(1-\mathscr{I}_{1} \tilde{\mathscr{M}}_{x}^{\beta}(y)\right) \vee\left(\mathscr{I}_{\mathscr{A}}(y) \vee \mathscr{I}_{\mathscr{B}}(y)\right)\right), \vee_{y \in \Omega}\left(\mathscr{T}_{1} \tilde{\mathscr{M}}_{x}^{\beta}(y) \wedge\left(\mathscr{F}_{\mathscr{A}}(y) \vee \mathscr{F}_{\mathscr{B}}(y)\right)\right)\right\rangle\right\} \\
& =\left\{\left\langlex, \wedge_{y \in \Omega}\left(\left(\mathscr{F}_{1} \tilde{\mathscr{M}}_{x}^{\beta}(y) \vee \mathscr{T}_{\mathscr{A}}(y)\right) \wedge\left(\mathscr{F}_{1} \tilde{\mathscr{M}}_{x}^{\beta}(y) \vee \mathscr{T}_{\mathscr{B}}(y)\right)\right), \wedge_{y \in \Omega}\left(\left(\left(1-\mathscr{I}_{{ }_{1}} \tilde{\mathbb{M}}_{x}^{\beta}(y)\right) \vee \mathscr{I}_{\mathscr{A}}(y)\right) \vee\left(\left(1-\mathscr{I}_{1} \tilde{\mathscr{M}}_{x}^{\beta}(y)\right) \vee \mathscr{I}_{\mathscr{B}}(y)\right)\right),\right.\right. \\
& \left.\left.\vee_{y \in \Omega}\left(\left(\mathscr{T}_{{ }_{1} \tilde{M}_{x}^{\beta}}(y) \wedge \mathscr{F}_{\mathscr{A}}(y)\right) \vee\left(\mathscr{T}_{{ }_{1} \tilde{\mathscr{M}}_{x}^{\beta}}(y) \wedge \mathscr{F}_{\mathscr{B}}(y)\right)\right)\right\rangle\right\} \\
& =\mathscr{L}_{2}^{1}(\mathscr{A}) \cap \mathscr{L}_{2}^{1}(\mathscr{B}) \text {. }
\end{aligned}
$$

(SVNL4): since $\mathscr{A} \cup \mathscr{B} \supseteq \mathscr{A}$, then, by (SVNL2), we have $\mathscr{L}_{2}^{1}(\mathscr{A} \cup \mathscr{B}) \supseteq \mathscr{L}_{2}^{1}(\mathscr{A})$. Similarly, $\mathscr{A} \cup \mathscr{B} \supseteq \mathscr{B} ;$ then, by (SVNL2), we have $\mathscr{L}_{2}^{1}(\mathscr{A} \cup \mathscr{B}) \supseteq \mathscr{L}_{2}^{1}(\mathscr{B})$. Thus, $\mathscr{L}_{2}^{1}(\mathscr{A} \cup \mathscr{B}) \supseteq \mathscr{L}_{2}^{1}(\mathscr{A}) \cup \mathscr{L}_{2}^{1}(\mathscr{B})$.

Now, we proceed to explain some relationships among these models.

Proposition 4. Let $(\Omega, \widetilde{\Gamma})$ be a 1-SVN $\beta C A S$ and $\mathscr{A} \in \operatorname{SVN}(\Omega)$. Then, we have the following properties:
(1) $\mathscr{L}_{4}^{1}(\mathscr{A}) \leq \mathscr{L}_{2}^{1}(\mathscr{A}) \leq \mathscr{L}_{3}^{1}(\mathscr{A})$
(2) $\mathscr{L}_{4}^{1}(\mathscr{A}) \leq \mathscr{L}_{1}^{1}(\mathscr{A}) \leq \mathscr{L}_{3}^{1}(\mathscr{A})$
(3) $\mathscr{U}_{3}^{1}(\mathscr{A}) \leq \mathscr{U}_{2}^{1}(\mathscr{A}) \leq \mathscr{L}_{4}^{1}(\mathscr{A})$
(4) $\mathscr{U}_{3}^{1}(\mathscr{A}) \leq \mathscr{U}_{1}^{1}(\mathscr{A}) \leq \mathscr{U}_{4}^{1}(\mathscr{A})$

Proof. The proof is clear from Definition 8.

Proposition 5. Let $(\Omega, \widetilde{\Gamma})$ be a 1-SVN $\beta C A S$ and $\mathscr{A} \in \operatorname{SVN}(\Omega)$. Then, we have the following properties:
(1) $\mathscr{L}_{3}^{1}(\mathscr{A}) \geq \mathscr{L}_{1}^{1}(\mathscr{A}) \cup \mathscr{L}_{2}^{1}(\mathscr{A})$
(2) $\mathscr{U}_{3}^{1}(\mathscr{A}) \leq \mathscr{U}_{1}^{1}(\mathscr{A}) \cap \mathscr{U}_{2}^{1}(\mathscr{A})$
(3) $\mathscr{L}_{4}^{1}(\mathscr{A}) \leq \mathscr{L}_{1}^{1}(\mathscr{A}) \cap \mathscr{L}_{2}^{1}(\mathscr{A})$
(4) $\mathscr{U}_{4}^{1}(\mathscr{A}) \geq \mathscr{U}_{1}^{1}(\mathscr{A}) \cup \mathcal{U}_{2}^{1}(\mathscr{A})$

Proof (straightforward)

\section{Type-2 SVN Complementary $\beta$-Neighborhood and Three New Kinds of Type-2 SVN $\beta$-CRS}

Definition 9. Let $(\Omega, \widetilde{\Gamma})$ be a 2-SVN $\beta$ CAS with $\widetilde{\Gamma}=\left\{\widetilde{C}_{1}, \widetilde{C}_{2}, \ldots, \widetilde{C}_{m}\right\}$, for some $\beta=\langle a, b, c\rangle$. Then, for each $x \in \Omega$, define the type- 2 SVN complementary $\beta$-neighborhood of $x$ as follows:

$$
{ }_{2} \widetilde{M}_{x}^{\beta}(y)={ }_{2} \widetilde{\mathcal{N}}_{y}^{\beta}(x), \quad \forall y \in \Omega .
$$

Example 3. Consider Example $1, \beta=\langle 0.5,0.1,0.8\rangle$ and $\mathscr{A}=$ $\left((0.6,0.3,0.5) / x_{1}\right)+\left((0.4,0.5,0.1) / x_{2}\right)+((0.3,0.2,0.6) /$ $\left.x_{3}\right)+\left((0.5,0.3,0.4) / x_{4}\right)+\left((0.7,0.2,0.3) / x_{5}\right)$. Then, the values of type- 2 SVN $\beta$-neighborhood are seen in Table 6:

$$
\begin{aligned}
\widetilde{2}_{2} \mathcal{N}_{x_{1}}^{\beta} & =\widetilde{C}_{1} \sqcap \widetilde{C}_{2}, \\
\widetilde{2}_{2}^{\beta} & =\widetilde{C}_{x_{2}} \sqcap \widetilde{C}_{2} \sqcap \widetilde{C}_{4}, \\
\widetilde{N}^{\beta} \mathcal{N}_{x_{3}}^{\beta} & =\widetilde{C}_{3} \sqcap \widetilde{C}_{4}, \\
\widetilde{2}_{{ }^{2}}^{\beta} & =\widetilde{C}_{x_{4}} \sqcap \widetilde{C}_{4}, \\
\widetilde{2}_{{ }^{2}}^{\beta} & =\widetilde{C}_{x_{5}} \sqcap \widetilde{C}_{3} \sqcap \widetilde{C}_{4} .
\end{aligned}
$$

Also, we compute type-2 SVN complementary $\beta$-neighborhood as in Table 7 .

Thus, ${ }_{\beta}$ we can merge ${ }_{2} \dot{\mathscr{M}}_{x}^{\beta}$ and ${ }_{2} \widetilde{M}_{x}^{\beta}$ and calculate ${ }_{2} \widetilde{\mathcal{N}}_{x}^{\beta} \Pi_{2} \widetilde{\mathscr{M}}_{x}^{\beta}$ as Table 8 .

Furthermore, we can calculate ${ }_{2} \tilde{\mathcal{N}}_{x}^{\beta} \sqcup_{2} \widetilde{\mathscr{M}}_{x}^{\beta}$, as set in Table 9.

Proposition 6. Let $(\Omega, \widetilde{\Gamma})$ be a 2-SVN $\beta C A S$, for some $\beta=$ $\langle a, b, c\rangle$ and for each $x, y, z \in \Omega$. Then, the following statements hold:

(1) ${ }_{2} \widetilde{M}_{x}^{\beta}(x) \geqslant \beta$

(2) If ${ }_{2} \mathscr{M}_{x}^{\beta}(y) \geqslant \beta$ and $\widetilde{\mathscr{M}}_{y}^{\beta}(z) \geqslant \beta$, then ${ }_{2} \widetilde{\mathscr{M}}_{x}^{\beta}(z) \geqslant \beta$

(3) $0 \prec \beta_{1} \preccurlyeq \beta_{2} \preccurlyeq \beta$, then ${ }_{2} \mathscr{M}_{x}^{\beta_{1}} \varpi_{2} \widetilde{M}_{x}^{\beta_{2}}$

Proof

(1) follows directly from Definitions 5 and 9.

(2) Since ${ }_{2} \widetilde{\mathscr{M}}_{x}^{\beta}(y) \geqslant \beta$, then ${ }_{2} \widetilde{\mathcal{N}}_{y}^{\beta}(x) \geqslant \beta$. If $\widetilde{C}_{i}(x) \geqslant \beta$, then $\widetilde{C}_{i}(y) \geqslant \beta$, and since ${ }_{2} \widetilde{M}_{y}^{\beta}(z) \geqslant \beta$, then ${ }_{2} \widetilde{N}_{z}^{\beta}(y) \geqslant \beta$. If $\widetilde{C}_{i}(y) \geqslant \beta$, then $\widetilde{C}_{i}(z) \geqslant \beta$. Therefore, ${ }_{2} \widetilde{M}_{x}^{\beta}(z) \geqslant \beta$.

(3) For each $x \in \Omega, 0<\beta_{1} \prec \beta_{2} \prec \beta$, then $\Pi\left\{\widetilde{C}_{i} \in \widetilde{\Gamma}\right.$ : $\left.\widetilde{C}_{i}(x) \geqslant \beta_{1}\right\} \sqsupseteq \sqcap\left\{\widetilde{C}_{i} \in \widetilde{\Gamma}: \widetilde{C}_{i}(x) \geqslant \beta_{2}\right\}$. Thus, by Definition 9, we have ${ }_{2} \widetilde{M}_{x}^{\beta_{1}} \sqsubseteq_{2} \widetilde{M}_{x}^{\beta_{2}}$. 
TABle $6:{ }_{2} \widetilde{N}_{x_{i}}^{\beta}, \forall i=1,2, \ldots, 5$.

\begin{tabular}{cccccc}
\hline & $x_{1}$ & $x_{2}$ & $x_{3}$ & $x_{4}$ & $x_{5}$ \\
\hline$\widetilde{2}^{\beta}$ & $\langle 0.6,0.2,0.5\rangle$ & $\langle 0.5,0.2,0.8\rangle$ & $\langle 0.2,0.3,0.6\rangle$ & $\langle 0.4,0.1,0.7\rangle$ & $\langle 0.3,0.2,0.6\rangle$ \\
${ }_{x_{1}}^{\beta}$ & $\langle 0.1,0.2,0.6\rangle$ & $\langle 0.5,0.1,0.8\rangle$ & $\langle 0.2,0.3,0.6\rangle$ & $\langle 0.4,0.1,0.7\rangle$ & $\langle 0.3,0.1,0.6\rangle$ \\
${ }^{2} \mathcal{N}_{x_{2}}^{\beta}$ & $\langle 0.1,0.1,0.6\rangle$ & $\langle 0.4,0.1,0.7\rangle$ & $\langle 0.5,0.2,0.4\rangle$ & $\langle 0.3,0.3,0.5\rangle$ & $\langle 0.6,0.1,0.5\rangle$ \\
${ }^{2} \mathcal{N}_{x_{3}}^{\beta}$ & $\langle 0.1,0.2,0.6\rangle$ & $\langle 0.5,0.1,0.7\rangle$ & $\langle 0.4,0.3,0.4\rangle$ & $\langle 0.5,0.1,0.7\rangle$ & $\langle 0.3,0.1,0.6\rangle$ \\
$\widetilde{ }^{2} \mathcal{N}_{x_{4}}^{\beta}$ & $\langle 0.1,0.1,0.6\rangle$ & $\langle 0.4,0.1,0.8\rangle$ & $\langle 0.2,0.2,0.6\rangle$ & $\langle 0.3,0.3,0.7\rangle$ & $\langle 0.6,0.1,0.5\rangle$ \\
$\widetilde{ }_{2}^{\beta} \mathcal{N}_{x_{5}}^{\beta}$ & & & & & \\
\hline
\end{tabular}

TABLE 7: $\widetilde{M}_{x_{i}}^{\beta}, \forall i=1,2, \ldots, 5$.

\begin{tabular}{|c|c|c|c|c|c|}
\hline & $x_{1}$ & $x_{2}$ & $x_{3}$ & $x_{4}$ & $x_{5}$ \\
\hline${ }_{2} \widetilde{M}_{x_{1}}^{\beta}$ & $\langle 0.5,0.8,0.6\rangle$ & $\langle 0.8,0.8,0.5\rangle$ & $\langle 0.6,0.7,0.2\rangle$ & $\langle 0.7,0.9,0.4\rangle$ & $\langle 0.6,0.8,0.3\rangle$ \\
\hline${ }_{2} \widetilde{M}_{x_{2}}^{\beta}$ & $\langle 0.6,0.8,0.1\rangle$ & $\langle 0.8,0.9,0.5\rangle$ & $\langle 0.6,0.7,0.2\rangle$ & $\langle 0.7,0.9,0.4\rangle$ & $\langle 0.6,0.9,0.3\rangle$ \\
\hline${ }_{2} \widetilde{M}_{x_{3}}^{\beta}$ & $\langle 0.6,0.9,0.1\rangle$ & $\langle 0.7,0.9,0.4\rangle$ & $\langle 0.4,0.8,0.5\rangle$ & $\langle 0.5,0.7,0.3\rangle$ & $\langle 0.5,0.9,0.6\rangle$ \\
\hline${ }_{2} \widetilde{M}_{x_{4}}^{\beta_{3}}$ & $\langle 0.6,0.8,0.1\rangle$ & $\langle 0.7,0.9,0.5\rangle$ & $\langle 0.4,0.7,0.4\rangle$ & $\langle 0.7,0.9,0.5\rangle$ & $\langle 0.6,0.9,0.3\rangle$ \\
\hline${ }_{2} \widetilde{M}_{x_{5}}^{\beta}$ & $\langle 0.6,0.9,0.1\rangle$ & $\langle 0.8,0.9,0.4\rangle$ & $\langle 0.6,0.8,0.2\rangle$ & $\langle 0.7,0.7,0.3\rangle$ & $\langle 0.5,0.9,0.6\rangle$ \\
\hline
\end{tabular}

TABLe 8: ${ }_{2} \widetilde{N}_{x_{i} \Pi_{2}}^{\beta} \widetilde{\mathscr{M}}_{x_{i}}^{\beta}, \forall i=1,2, \ldots, 5$.

\begin{tabular}{|c|c|c|c|c|c|}
\hline & $x_{1}$ & $x_{2}$ & $x_{3}$ & $x_{4}$ & $x_{5}$ \\
\hline${ }_{2} \widetilde{N}_{x_{1} \Pi_{2}}^{\beta} \widetilde{M}_{x_{1}}^{\beta}$ & $\langle 0.5,0.2,0.6\rangle$ & $\langle 0.5,0.2,0.8\rangle$ & $\langle 0.2,0.3,0.6\rangle$ & $\langle 0.4,0.1,0.7\rangle$ & $\langle 0.3,0.2,0.6\rangle$ \\
\hline$\widetilde{\mathcal{N}}_{x_{2}}^{\beta} \tilde{\Pi}_{2} \widetilde{M}_{x_{2}}^{\beta}$ & $\langle 0.1,0.2,0.6\rangle$ & $\langle 0.5,0.1,0.8\rangle$ & $\langle 0.2,0.3,0.6\rangle$ & $\langle 0.4,0.1,0.7\rangle$ & $\langle 0.3,0.1,0.6\rangle$ \\
\hline$\widetilde{N}_{2} \widetilde{N}_{x_{3}}^{\beta} \Pi_{2} \widetilde{M}_{x_{3}}^{\beta}$ & $\langle 0.1,0.1,0.6\rangle$ & $\langle 0.4,0.1,0.7\rangle$ & $\langle 0.4,0.2,0.5\rangle$ & $\langle 0.3,0.3,0.5\rangle$ & $\langle 0.5,0.1,0.6\rangle$ \\
\hline$\widetilde{2}_{2} \mathcal{N}_{x_{4}}^{\beta} \Pi_{2} \widetilde{M}_{x_{4}}^{\beta}$ & $\langle 0.1,0.2,0.6\rangle$ & $\langle 0.5,0.1,0.7\rangle$ & $\langle 0.4,0.3,0.4\rangle$ & $\langle 0.5,0.1,0.7\rangle$ & $\langle 0.3,0.1,0.6\rangle$ \\
\hline$\widetilde{ }_{2} \widetilde{N}_{x_{5}}^{\beta} \Pi_{2} \widetilde{M}_{x_{5}}^{\beta}$ & $\langle 0.1,0.1,0.6\rangle$ & $\langle 0.4,0.1,0.8\rangle$ & $\langle 0.2,0.2,0.6\rangle$ & $\langle 0.3,0.3,0.7\rangle$ & $\langle 0.5,0.1,0.6\rangle$ \\
\hline
\end{tabular}

TABLE 9: $\widetilde{\mathscr{N}}_{x_{i}}^{\beta} \sqcup_{2} \widetilde{\mathscr{M}}_{x_{i}}^{\beta}, \forall i=1,2, \ldots, 5$.

\begin{tabular}{|c|c|c|c|c|c|}
\hline & $x_{1}$ & $x_{2}$ & $x_{3}$ & $x_{4}$ & $x_{5}$ \\
\hline$\widetilde{\mathcal{N}}_{x_{1}}^{\beta} \sqcup_{2} \widetilde{M}_{x_{1}}^{\beta}$ & $\langle 0.6,0.8,0.5\rangle$ & $\langle 0.8,0.8,0.5\rangle$ & $\langle 0.6,0.7,0.2\rangle$ & $\langle 0.7,0.9,0.4\rangle$ & $\langle 0.6,0.8,0.3\rangle$ \\
\hline$\widetilde{\mathcal{N}}_{x_{2}}^{\beta} \sqcup_{2} \widetilde{M}_{x_{2}}^{\beta}$ & $\langle 0.6,0.8,0.1\rangle$ & $\langle 0.8,0.9,0.5\rangle$ & $\langle 0.6,0.7,0.2\rangle$ & $\langle 0.7,0.9,0.4\rangle$ & $\langle 0.6,0.9,0.3\rangle$ \\
\hline$\widetilde{N}_{2}^{\beta_{x_{3}}^{\beta}} \mathrm{J}_{2} \widetilde{M}_{x_{3}}^{\beta^{2}}$ & $\langle 0.6,0.9,0.1\rangle$ & $\langle 0.7,0.9,0.4\rangle$ & $\langle 0.5,0.8,0.4\rangle$ & $\langle 0.5,0.7,0.3\rangle$ & $\langle 0.6,0.9,0.5\rangle$ \\
\hline$\widetilde{N}_{2}^{\beta}{ }_{x_{4}}^{\beta} \widetilde{M}_{x_{4}}^{\beta}$ & $\langle 0.6,0.8,0.1\rangle$ & $\langle 0.7,0.9,0.5\rangle$ & $\langle 0.4,0.7,0.4\rangle$ & $\langle 0.7,0.9,0.5\rangle$ & $\langle 0.6,0.9,0.3\rangle$ \\
\hline$\widetilde{2}_{2}^{\beta}{ }_{x_{5}}^{\beta} \sqcup_{2} \widetilde{M}_{x_{5}}^{\beta}$ & $\langle 0.6,0.9,0.1\rangle$ & $\langle 0.8,0.9,0.4\rangle$ & $\langle 0.6,0.8,0.2\rangle$ & $\langle 0.7,0.7,0.3\rangle$ & $\langle 0.6,0.9,0.5\rangle$ \\
\hline
\end{tabular}

Proposition 7. Let $(\Omega, \widetilde{\Gamma})$ be a 1-SVN $\beta C A S$, for some $\beta=\langle a, b, c\rangle$. For each $x, y \in \Omega,{ }_{2} \widetilde{\mathscr{M}}_{x}^{\beta}(y) \geqslant \beta \Leftrightarrow{ }_{2} \widetilde{\mathscr{M}}_{y}^{\beta} \Xi_{2} \widetilde{\mathscr{M}}_{x}^{\beta}$.

Proof. Let $\quad \widetilde{\mathscr{M}}_{x}^{\beta}(y) \geqslant \beta, \quad \mathscr{T}_{{ }_{2}} \widetilde{M}_{x}^{\beta}(y)=\mathscr{T} \sqcap \widetilde{C}_{i}(y)=$ $\wedge \mathscr{T}_{\widetilde{C}_{i}}(y) \geq a, \mathscr{I} \widetilde{\mathscr{M}}_{x}^{\beta}(y)=\mathscr{I}_{\Pi} \widetilde{C}_{i}(y)=\vee \mathscr{I}_{\widetilde{C}_{i}}(y) \geq b, \quad$ and $\mathscr{F}_{2} \tilde{\mathscr{M}}_{x}^{\beta}(y)=\mathscr{F} \sqcap \widetilde{C}_{i}(y)=\vee \mathscr{F}_{\widetilde{C}_{i}}(y) \leq c . \quad$ Then, $\quad\left\{\widetilde{C}_{i} \in \widetilde{\Gamma}:\right.$ $\left.\mathscr{T}_{\widetilde{C}_{i}}(x) \geq a, \mathscr{I}_{\widetilde{C}_{i}}(x) \geq b, \mathscr{F}_{\widetilde{C}_{i}}(x) \leq c\right\} \quad \subseteq\left\{\widetilde{C}_{i} \in \widetilde{\Gamma}: \mathscr{T}_{\widetilde{C}_{i}}(y) \geq\right.$ $\left.a, \mathscr{I}_{\widetilde{C}_{i}}(y) \geq b, \mathscr{F}_{\widetilde{C}_{i}}(y) \leq c\right\}$ Thus, $\widetilde{2}_{2}^{\beta} \widetilde{M}_{y} \widetilde{\mathscr{M}}_{x}^{\beta}$. On the contrary, let $\widetilde{\mathscr{M}}_{y}^{\beta} \sqsubseteq_{2} \widetilde{\mathscr{M}}_{x}^{\beta}$. Then, $\mathscr{T}_{{ }_{2}} \widetilde{M}_{x}^{\beta}(y) \geq \mathscr{T}_{{ }_{2}} \widetilde{M}_{y}^{\beta}(y) \geq a$,
$\mathscr{I} \tilde{\mathscr{M}}_{x}^{\beta}(y) \geq \mathscr{I} \widetilde{\sim}_{\beta}(y) \geq b$, and $\mathscr{F}{ }_{2} \tilde{\mathscr{M}}_{x}^{\beta}(y) \leq \mathscr{F}{\widetilde{\mathscr{M}_{y}}}_{\beta}^{\beta}(y) \leq c$. Hence, ${ }_{2} \widetilde{M}_{x}^{\beta}\left({ }^{2}\right) \geqslant \beta$.

Here, we construct three new types of $2-1-S V N \beta C R S s$ based on Definitions 5 and 9 as seen below.

Definition 10. Consider $(\Omega, \widetilde{\Gamma})$ be a 2 -SVN $\beta$ CAS with $\widetilde{\Gamma}=$ $\left\{\widetilde{C}_{1}, \widetilde{C}_{2}, \ldots, \widetilde{C}_{m}\right\}$ for some $\beta=\langle a, b, c\rangle$. For each $x \in \Omega$ and $\mathscr{A} \in \operatorname{SVN}(\Omega)$, then we have the following paradigms.

Paradigm 1: The second type of Type-2 SVN lower approximation (2-2-SVNLA) $\mathscr{L}_{2}^{2}(\mathscr{A})$ and the second 
type of Type-2 SVN upper approximation (2-2-

SVNUA) $\mathscr{U}_{2}^{2}(\mathscr{A})$ are found as follows:

$$
\begin{aligned}
& \mathscr{L}_{2}^{2}(\mathscr{A})=\left\{\left\langle x, \wedge_{y \in \Omega}\left(\mathscr{F}_{2} \tilde{\mathscr{M}}_{x}^{\beta}(y) \vee \mathscr{T}_{\mathscr{A}}(y)\right), \wedge_{y \in \Omega}\left(\left(1-\mathscr{I}_{2} \tilde{\mathscr{M}}_{x}^{\beta}(y)\right) \vee \mathscr{F}_{\mathscr{A}}(y)\right), \vee_{y \in \Omega}\left(\mathscr{T}_{2} \tilde{\mathscr{M}}_{x}^{\beta}(y) \wedge \mathscr{F}_{\mathscr{A}}(y)\right)\right\rangle\right\}, \\
& \mathscr{U}_{2}^{2}(\mathscr{A})=\left\{\left\langle x, \vee_{y \in \Omega}\left(\mathscr{T}_{2} \tilde{\mathscr{M}}_{x}^{\beta}(y) \wedge \mathscr{T}_{\mathscr{A}}(y)\right), \vee_{y \in \Omega}\left(\mathscr{I}_{2} \tilde{\mathscr{M}}_{x}^{\beta}(y) \wedge \mathscr{F}_{\mathscr{A}}(y)\right), \wedge_{y \in \Omega}\left(\mathscr{F}_{2} \tilde{\mathscr{M}}_{x}^{\beta}(y) \vee \mathscr{F}_{\mathscr{A}}(y)\right)\right\rangle\right\} .
\end{aligned}
$$

If $\mathscr{L}_{2}^{2}(\mathscr{A}) \neq \mathscr{U}_{2}^{2}(\mathscr{A})$, then $\mathscr{A}$ is called the second type of Type-2 SVN $\beta$-covering rough sets (briefly, 2-2SVN $\beta$ CRSs)

Paradigm 2: the third type of Type-2 SVN lower approximation (3-2-SVNLA) $\mathscr{L}_{3}^{2}(\mathscr{A})$ and the third type of Type-2 SVN upper approximation (3-2-SVNUA) $\mathscr{U}_{3}^{2}(\mathscr{A})$ are introduced as follows:

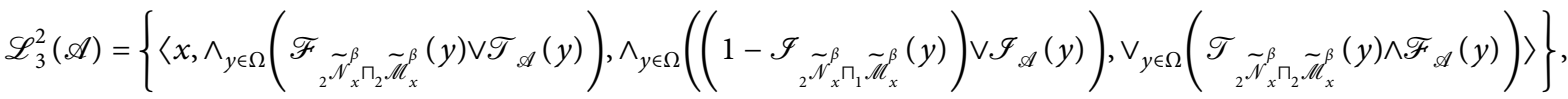

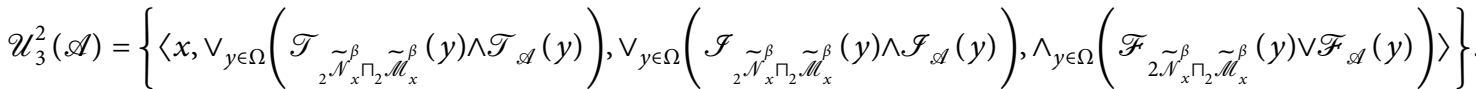

If $\mathscr{L}_{3}^{2}(\mathscr{A}) \neq \mathscr{U}_{3}^{2}(\mathscr{A})$, then $\mathscr{A}$ is called the third type of Type-2 SVN $\beta$-covering rough sets (briefly, 3-2SVN $\beta$ CRSs).
Paradigm 3: the fourth type of Type-2 SVN lower approximation (4-2-SVNLA) $\mathscr{L}_{4}^{2}(\mathscr{A})$ and the fourth type of Type-2 SVN upper approximation (4-2SVNUA) $\mathcal{U}_{4}^{2}(\mathscr{A})$ are proposed as follows:

$$
\begin{aligned}
& \mathscr{L}_{4}^{2}(\mathscr{A})=\left\{\left\langle x, \wedge_{y \in \Omega}\left(\mathscr{F}_{2} \tilde{\mathcal{N}} x^{\beta} \sqcup_{2} \widetilde{\mathscr{M}}_{x}^{\beta}(y) \vee \mathscr{T}_{\mathscr{A}}(y)\right), \wedge_{y \in \Omega}\left(\left(1-\mathscr{I}_{2} \tilde{\mathcal{N}} x^{\beta} \sqcup_{2} \widetilde{\mathbb{M}}_{x}^{\beta}(y)\right) \vee \mathscr{I}_{\mathscr{A}}(y)\right), \vee_{y \in \Omega}\left(\mathscr{T}_{2} \tilde{\mathcal{N}} x^{\beta} \sqcup_{2} \widetilde{\mathbb{M}}_{x}^{\beta}(y) \wedge \mathscr{F}_{\mathscr{A}}(y)\right)\right\rangle\right\},
\end{aligned}
$$

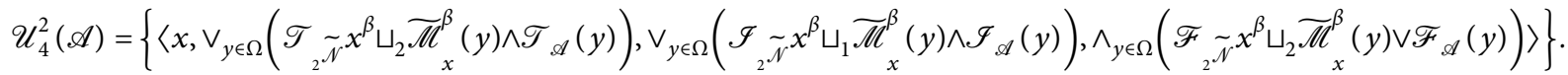

If $\mathscr{L}_{4}^{2}(\mathscr{A}) \neq \mathscr{U}_{4}^{2}(\mathscr{A})$, then $\mathscr{A}$ is called the fourth type of Type-2 SVN $\beta$-covering rough sets (briefly, 4-2SVN $\beta$ CRSs).
Example 4. Consider Example 1 if $\beta=\langle 0.5,0.1,0.8\rangle$ and $\mathscr{A}=\left((0.6,0.3,0.5) / x_{1}\right)+\left((0.4,0.5,0.1) / x_{2}\right)+((0.3,0.2,0.6) /$ $\left.x_{3}\right)+\left((0.5,0.3,0.4) / x_{4}\right)+\left((0.7,0.2,0.3) / x_{5}\right)$; then, we have the following results:

$$
\begin{aligned}
& \mathscr{L}_{1}^{2}(\mathscr{A})=\left\{\left\langle x_{1}, 0.6,0.7,0.5\right\rangle,\left\langle x_{2}, 0.6,0.7,0.4\right\rangle,\left\langle x_{3}, 0.4,0.7,0.5\right\rangle,\left\langle x_{4}, 0.4,0.7,0.4\right\rangle,\left\langle x_{5}, 0.6,0.7,0.3\right\rangle\right\}, \\
& \mathscr{U}_{1}^{2}(\mathscr{A})=\left\{\left\langle x_{1}, 0.6,0.2,0.5\right\rangle,\left\langle x_{2}, 0.4,0.2,0.6\right\rangle,\left\langle x_{3}, 0.6,0.3,0.5\right\rangle,\left\langle x_{4}, 0.5,0.2,0.6\right\rangle,\left\langle x_{5}, 0.6,0.3,0.5\right\rangle\right\}, \\
& \mathscr{L}_{2}^{2}(\mathscr{A})=\left\{\left\langle x_{1}, 0.3,0.2,0.6\right\rangle,\left\langle x_{2}, 0.3,0.2,0.6\right\rangle,\left\langle x_{3}, 0.4,0.2,0.5\right\rangle,\left\langle x_{4}, 0.4,0.2,0.4\right\rangle,\left\langle x_{5}, 0.3,0.2,0.6\right\rangle\right\}, \\
& \mathscr{U}_{2}^{2}(\mathscr{A})=\left\{\left\langle x_{1}, 0.6,0.5,0.3\right\rangle,\left\langle x_{2}, 0.6,0.5,0.3\right\rangle,\left\langle x_{3}, 0.6,0.5,0.4\right\rangle,\left\langle x_{4}, 0.6,0.5,0.3\right\rangle,\left\langle x_{5}, 0.6,0.5,0.4\right\rangle\right\}, \\
& \mathscr{L}_{3}^{2}(\mathscr{A})=\left\{\left\langle x_{1}, 0.6,0.7,0.5\right\rangle,\left\langle x_{2}, 0.6,0.7,0.4\right\rangle,\left\langle x_{3}, 0.5,0.7,0.4\right\rangle,\left\langle x_{4}, 0.4,0.7,0.3\right\rangle,\left\langle x_{5}, 0.6,0.7,0.3\right\rangle\right\}, \\
& \mathscr{U}_{3}^{2}(\mathscr{A})=\left\{\left\langle x_{1}, 0.5,0.2,0.6\right\rangle,\left\langle x_{2}, 0.4,0.2,0.6\right\rangle,\left\langle x_{3}, 0.5,0.3,0.5\right\rangle,\left\langle x_{4}, 0.5,0.2,0.6\right\rangle,\left\langle x_{5}, 0.5,0.3,0.6\right\rangle\right\}, \\
& \mathscr{L}_{4}^{2}(\mathscr{A})=\left\{\left\langle x_{1}, 0.3,0.2,0.6\right\rangle,\left\langle x_{2}, 0.3,0.2,0.6\right\rangle,\left\langle x_{3}, 0.4,0.2,0.5\right\rangle,\left\langle x_{4}, 0.4,0.2,0.5\right\rangle,\left\langle x_{5}, 0.3,0.2,0.6\right\rangle\right\}, \\
& \mathscr{U}_{4}^{2}(\mathscr{A})=\left\{\left\langle x_{1}, 0.6,0.5,0.3\right\rangle,\left\langle x_{2}, 0.6,0.5,0.3\right\rangle,\left\langle x_{3}, 0.6,0.5,0.4\right\rangle,\left\langle x_{4}, 0.6,0.5,0.3\right\rangle,\left\langle x_{5}, 0.6,0.5,0.4\right\rangle\right\} .
\end{aligned}
$$


In the following, we will propose Proposition 8 for the 2 2 -SVN $\beta$ CRS model; also, it fulfills in case of the 3-2SVN $\beta$ CRS and the 4-2-SVN $\beta$ CRS models.

Proposition 8. Let $(\Omega, \widetilde{\Gamma})$ be a 1-SVN $\beta C A S$, for some $\beta=\langle a, b, c\rangle$. For each $x, y, z \in \Omega$ and $\mathscr{A}, \mathscr{B} \in \operatorname{SVN}(\Omega)$, then the following statements hold:

(1) $(S V N L 1): \mathscr{L}_{2}^{2}\left(\mathscr{A}^{c}\right)=\left(\mathscr{U}_{2}^{2}(\mathscr{A})\right)^{c}$. (SVNU1): $\mathscr{U}_{2}^{2}\left(\mathscr{A}^{c}\right)=\left(\mathscr{L}_{2}^{2}(\mathscr{A})\right)^{c}$.

(2) If $\mathscr{A} \subseteq \mathscr{B}$, then (SVNL2): $\mathscr{L}_{2}^{2}(\mathscr{A}) \subseteq \mathscr{L}_{2}^{2}(\mathscr{B})$. (SVNU2): $\mathscr{U}_{2}^{2}(\mathscr{A}) \subseteq \mathcal{U}_{2}^{2}(\mathscr{B})$.
(3) (SVNL3): $\mathscr{L}_{2}^{2}(\mathscr{A} \cap \mathscr{B})=\mathscr{L}_{2}^{2}(\mathscr{A}) \cap \mathscr{L}_{2}^{2}(\mathscr{B})$. (SVNU3): $\mathscr{U}_{2}^{2}(\mathscr{A} \cap \mathscr{B}) \subseteq \mathcal{U}_{2}^{2}(\mathscr{A}) \cap \mathcal{U}_{2}^{2}(\mathscr{B})$.

(4) (SVNL4): $\mathscr{L}_{2}^{2}(\mathscr{A} \cup \mathscr{B}) \supseteq \mathscr{L}_{2}^{2}(\mathscr{A}) \cup \mathscr{L}_{2}^{2}(\mathscr{B})$. (SVNU4): $\mathscr{U}_{2}^{2}(\mathscr{A} \cap \mathscr{B})=\mathscr{U}_{2}^{2}(\mathscr{A}) \cap \mathcal{U}_{2}^{1}(\mathscr{B})$.

(5) (SVNL5): $\mathscr{L}_{2}^{2}(\Omega)=\Omega$.

(SVNU5): $\mathscr{U}_{2}^{2}(\varnothing)=\varnothing$.

Proof. We shall only prove (SVNL1), (SVNL2), (SVNL3), and (SVNL4).

(SVNL1):

$$
\begin{aligned}
\mathscr{L}_{2}^{2}\left(\mathscr{A}^{c}\right) & =\left\{\left\langle x, \wedge_{y \in \Omega}\left(\mathscr{F}_{2 \tilde{\mathscr{M}}_{x}^{\beta}}(y) \vee \mathscr{T}_{\mathscr{A}^{c}}(y)\right), \wedge_{y \in \Omega}\left(\left(1-\mathscr{I}_{2 \tilde{\mathscr{M}}_{x}^{\beta}}(y)\right) \vee \mathscr{I}_{\mathscr{A}^{c}}(y)\right), \vee_{y \in \Omega}\left(\mathscr{T}_{2 \tilde{\mathscr{M}}_{x}^{\beta}}(y) \wedge \mathscr{F}_{\mathscr{A}^{c}}(y)\right)\right\rangle\right\} \\
& =\left\{\left\langle x, \wedge_{y \in \Omega}\left(\mathscr{F}_{2} \tilde{\mathscr{M}}_{x}^{\beta}(y) \vee \mathscr{F}_{\mathscr{A}}(y)\right), \wedge_{y \in \Omega}\left(\left(1-\mathscr{I}_{2 \tilde{\mathscr{M}}_{x}^{\beta}}(y)\right) \vee\left(1-\mathscr{I}_{\mathscr{A}}(y)\right)\right), \vee_{y \in \Omega}\left(\mathscr{T}_{2 \tilde{\mathscr{M}}_{x}^{\beta}}(y) \wedge \mathscr{T}_{\mathscr{A}}(y)\right)\right\rangle\right\} \\
& =\left(\mathscr{U}_{2}^{2}(\mathscr{A})\right)^{c} .
\end{aligned}
$$

(SVNL2): let $\mathscr{A}, \mathscr{B} \in \operatorname{SVN}(\Omega)$ such that $\mathscr{A} \sqsubseteq \mathscr{B}$ (i.e., $\mathscr{T}_{\mathscr{A}} \leq \mathscr{T}_{\mathscr{B}}, \mathscr{I}_{\mathscr{B}} \leq \mathscr{I}_{\mathscr{A}}$ and $\left.\mathscr{F}_{\mathscr{B}} \leq \mathscr{F}_{\mathscr{A}}\right)$ and $x \in \Omega$. Then, we get the following result:

$$
\begin{aligned}
& \mathscr{L}_{2}^{2}(\mathscr{A})(x)=\left\{\left\langle x, \wedge_{y \in \Omega}\left(\mathscr{F}_{{ }_{2}} \tilde{\mathscr{M}}_{x}^{\beta}(y) \vee \mathscr{T}_{\mathscr{A}}(y)\right), \wedge_{y \in \Omega}\left(\left(1-\mathscr{I}_{{ }_{2}} \tilde{\mathscr{M}}_{x}^{\beta}(y)\right) \vee \mathscr{T}_{\mathscr{A}}(y)\right), \vee_{y \in \Omega}\left(\mathscr{T}_{{ }_{2}} \tilde{\mathscr{M}}_{x}^{\beta}(y) \wedge \mathscr{F}_{\mathscr{A}}(y)\right)\right\rangle\right\}, \\
& \mathscr{T}_{\mathscr{L}_{2}^{2}(\mathscr{A})}=\wedge_{y \in \Omega}\left(\mathscr{F}_{{ }_{2}} \tilde{M}_{x}^{\beta}(y) \vee \mathscr{T}_{\mathscr{A}}(y)\right) \leq \Lambda_{y \in \Omega}\left(\mathscr{F}_{{ }_{2}} \tilde{\mathscr{M}}_{x}^{\beta}(y) \vee \mathscr{T}_{\mathscr{B}}(y)\right)=\mathscr{T}_{\mathscr{L}_{2}^{2}(\mathscr{B})} \text {, } \\
& \mathscr{I}_{\mathscr{L}_{2}^{2}(\mathscr{A})}=\wedge_{y \in \Omega}\left(\left(1-\mathscr{I}_{{ }_{2} \tilde{\mathbb{M}}_{x}^{\beta}}(y)\right) \vee \mathscr{I}_{\mathscr{A}}(y)\right) \leq \Lambda_{y \in \Omega}\left(\left(1-\mathscr{I}_{2} \tilde{\mathscr{M}}_{x}^{\beta}(y)\right) \vee \mathscr{I}_{\mathscr{B}}(y)\right)=\mathscr{I}_{\mathscr{L}_{2}^{2}(\mathscr{B})}, \\
& \mathscr{F}_{\mathscr{L}_{2}^{2}(\mathscr{A})}=\vee_{y \in \Omega}\left(\mathscr{T}_{{ }_{2} \tilde{\mathscr{M}}_{x}^{\beta}}(y) \wedge \mathscr{F}_{\mathscr{A}}(y)\right) \geq \vee_{y \in \Omega}\left(\mathscr{T}_{2} \tilde{\mathscr{M}}_{x}^{\beta}(y) \wedge \mathscr{F}_{\mathscr{B}}(y)\right)=\mathscr{F}_{\mathscr{L}_{2}^{2}(\mathscr{B})} .
\end{aligned}
$$

Therefore, $\mathscr{L}_{2}^{2}(\mathscr{A}) \sqsubseteq \mathscr{L}_{2}^{2}(\mathscr{B})$.

(SVNL3): If $x \in \Omega$, then we have

$$
\begin{aligned}
& \mathscr{L}_{2}^{2}(\mathscr{A})(x)=\left\{\left\langle x, \wedge_{y \in \Omega}\left(\mathscr{F}_{2} \tilde{\mathscr{M}}_{x}^{\beta}(y) \vee \mathscr{T}_{\mathscr{A} \sqcap \mathscr{B}}(y)\right), \wedge_{y \in \Omega}\left(\left(1-\mathscr{I}_{2} \tilde{\mathscr{M}}_{x}^{\beta}(y)\right) \vee \mathscr{I}_{\mathscr{A} \sqcap \mathscr{B}}(y)\right), \vee_{y \in \Omega}\left(\mathscr{T}_{2} \tilde{\mathscr{M}}_{x}^{\beta}(y) \wedge \mathscr{F}_{\mathscr{A} \sqcap \mathscr{B}}(y)\right)\right\rangle\right\} \\
& =\left\{\left\langle x, \wedge_{y \in \Omega}\left(\mathscr{F}_{{ }_{2}} \tilde{\mathscr{M}}_{x}^{\beta}(y) \vee\left(\mathscr{T}_{\mathscr{A}}(y) \wedge \mathscr{T}_{\mathscr{B}}(y)\right)\right), \wedge_{y \in \Omega}\left(\left(1-\mathscr{I}_{{ }_{2}} \tilde{\mathscr{M}}_{x}^{\beta}(y)\right) \vee\left(\mathscr{I}_{\mathscr{A}}(y) \wedge \mathscr{F}_{\mathscr{B}}(y)\right)\right), \vee_{y \in \Omega}\left(\mathscr{T}_{2} \tilde{\mathscr{M}}_{x}^{\beta}(y) \wedge\left(\mathscr{F}_{\mathscr{A}}(y) \vee \mathscr{F}_{\mathscr{B}}(y)\right)\right)\right\rangle\right\} \\
& =\left\{\left\langlex, \wedge_{y \in \Omega}\left(\left(\mathscr{F}_{{ }_{2}} \tilde{\mathscr{M}}_{x}^{\beta}(y) \vee \mathscr{T}_{\mathscr{A}}(y)\right) \wedge\left(\mathscr{F}_{{ }_{2}} \tilde{\mathscr{M}}_{x}^{\beta}(y) \vee \mathscr{T}_{\mathscr{B}}(y)\right)\right), \wedge_{y \in \Omega}\left(\left(\left(1-\mathscr{I}_{{ }_{2}} \tilde{\mathscr{M}}_{x}^{\beta}(y)\right) \vee \mathscr{F}_{\mathscr{A}}(y)\right) \wedge\left(\left(1-\mathscr{I}_{{ }_{2} \tilde{M}_{x}^{\beta}}(y)\right) \vee \mathscr{I}_{\mathscr{B}}(y)\right)\right),\right.\right. \\
& \left.\left.\vee_{y \in \Omega}\left(\left(\mathscr{T}_{2} \tilde{\mathscr{M}}_{x}^{\beta}(y) \wedge\left(\mathscr{F}_{\mathscr{A}}(y)\right) \vee\left(\mathscr{T}_{2} \tilde{\mathscr{M}}_{x}^{\beta}(y) \wedge \mathscr{F}_{\mathscr{B}}(y)\right)\right)\right\rangle\right\rangle\right\} \\
& =\mathscr{L}_{2}^{2}(\mathscr{A}) \sqcap \mathscr{L}_{2}^{2}(\mathscr{B}) .
\end{aligned}
$$

(SVNL4): since $\mathscr{A} \sqcup \mathscr{B} \sqsupseteq \mathscr{A}$, then by SVNL2 we have $\mathscr{L}_{2}^{2}(\mathscr{A} \sqcup \mathscr{B}) \sqsupseteq \mathscr{L}_{2}^{2}(\mathscr{A})$. Similarly, $\mathscr{A} \sqcup \mathscr{B} \sqsupseteq \mathscr{B} ;$ then, by SVNL2, we have $\quad \mathscr{L}_{2}^{2}(\mathscr{A} \sqcup \mathscr{B}) \sqsupseteq \mathscr{L}_{2}^{2}(\mathscr{B})$. Thus, $\quad \mathscr{L}_{2}^{2}(\mathscr{A} \sqcup \mathscr{B}) \sqsupseteq$ $\mathscr{L}_{2}^{2}(\mathscr{A}) \sqcup \mathscr{L}_{2}^{2}(\mathscr{B})$. 
(SVNL5): since SVN universe is $\Omega=\langle x, 1,1,0\rangle$ and SVN empty set is $\varnothing=\langle x, 0,0,1\rangle$, then we have

$$
\begin{aligned}
\mathscr{L}_{2}^{2}(\Omega) & =\left\{\left\langle x, \wedge_{y \in \Omega}\left(\mathscr{F}_{2} \underset{{ }_{2}}{\tilde{M}_{x}^{\beta}}(y) \vee \mathscr{T}_{\Omega}(y)\right), \wedge_{y \in \Omega}\left(\left(1-\mathscr{I}_{2} \tilde{\mathscr{M}}_{x}^{\beta}(y)\right) \vee \mathscr{I}_{\Omega}(y)\right), \vee_{y \in \Omega}\left(\mathscr{T}_{2} \tilde{\mathscr{M}}_{x}^{\beta}(y) \wedge \mathscr{F}_{\Omega}(y)\right)\right\rangle\right\} \\
& =\left\{\left\langle x, \wedge_{y \in \Omega}\left(\mathscr{F}_{{ }_{2}} \tilde{\mathscr{M}}_{x}^{\beta}(y) \vee 1\right), \wedge_{y \in \Omega}\left(\left(1-\mathscr{I}_{{ }_{2}} \tilde{\mathscr{M}}_{x}^{\beta}(y)\right) \vee 1\right), \vee_{y \in \Omega}\left(\mathscr{T}_{2} \tilde{\mathscr{M}}_{x}^{\beta}(y) \wedge 0\right)\right\rangle\right\} \\
& =\{\langle x, 1,1,0\rangle\} \\
& =\Omega .
\end{aligned}
$$

In the following, we give some relationships among these models.

Proposition 9. Let $(\Omega, \widetilde{\Gamma})$ be a 2-SVN $\beta C A S$ and $\mathscr{A} \in S V N(\Omega)$. Then, we have the following properties:
(1) $\mathscr{L}_{4}^{2}(\mathscr{A}) \leqslant \mathscr{L}_{2}^{2}(\mathscr{A}) \leqslant \mathscr{L}_{3}^{2}(\mathscr{A})$
(2) $\mathscr{L}_{4}^{2}(\mathscr{A}) \leqslant \mathscr{L}_{1}^{2}(\mathscr{A}) \leqslant \mathscr{L}_{3}^{2}(\mathscr{A})$
(3) $\mathcal{U}_{3}^{2}(\mathscr{A}) \leqslant \mathcal{U}_{2}^{2}(\mathscr{A}) \leqslant \mathcal{U}_{4}^{2}(\mathscr{A})$
(4) $\mathcal{U}_{3}^{2}(\mathscr{A}) \leqslant \mathcal{U}_{1}^{2}(\mathscr{A}) \leqslant \mathscr{U}_{4}^{2}(\mathscr{A})$

Proof. The proof is clear from Definition 10.

Proposition 10. Let $(\Omega, \widetilde{\Gamma})$ be a 2-SVN $\beta C A S$ and $\mathscr{A} \in \operatorname{SVN}(\Omega)$. Then, we have the following properties:
(1) $\mathscr{L}_{3}^{2}(\mathscr{A}) \geqslant \mathscr{L}_{1}^{2}(\mathscr{A}) \sqcup \mathscr{L}_{2}^{2}(\mathscr{A})$
(2) $\mathcal{U}_{3}^{2}(\mathscr{A}) \preccurlyeq \mathcal{U}_{1}^{2}(\mathscr{A}) \sqcap \mathcal{U}_{2}^{2}(\mathscr{A})$
(3) $\mathscr{L}_{4}^{2}(\mathscr{A}) \geqslant \mathscr{L}_{1}^{2}(\mathscr{A}) \sqcap \mathscr{L}_{2}^{2}(\mathscr{A})$
(4) $\mathcal{U}_{4}^{2}(\mathscr{A}) \preccurlyeq \mathcal{U}_{1}^{2}(\mathscr{A}) \sqcup \mathcal{U}_{2}^{2}(\mathscr{A})$

Proof (clear)

\section{Decision-Making Approach to DM Based on SVN $\beta$ CRSs}

\subsection{Description and Process}

5.1.1. Method I. Assume that $\Omega=\left\{x_{r}: r=1, \ldots, k\right\}$ is the set of alternatives (patients), $m$ is main attributes (symptoms) (e.g., cough and fever) $V=\left\{y_{i}: i=1,2, \ldots, m\right\}$ of $A$ disease, $\widetilde{\mathscr{C}}_{i}\left(x_{r}\right)=\left\langle\mathscr{T}_{\tilde{C}_{i}}\left(x_{r}\right), \mathscr{I}_{\tilde{\mathscr{C}}_{i}}\left(x_{r}\right), \mathscr{F}_{\tilde{\mathscr{C}}_{i}}\left(x_{r}\right)\right\rangle$ indicates the symptom value for each patient which is known by a doctor $D$, for some $\beta=\langle a, b, c\rangle$, and $(\Omega, \widetilde{\Gamma})$ is a Type-1 SVN $\beta$-CRS, where $\mathscr{T}_{\tilde{C}}\left(x_{r}\right) \in[0,1]$ (i.e., the degree that doctor $D$ confirms the patient $x_{r}$ has symptom $\left.y_{i}\right), \mathscr{I}_{\tilde{\mathscr{C}}}\left(x_{r}\right) \in[0,1]$ (i.e., the degree that doctor $D$ is not sure if the patient $x_{r}$ has symptom $\left.y_{i}\right), \mathscr{F} \tilde{\mathscr{C}}_{i}\left(x_{r}\right) \in[0,1]$ (i.e., the degree that doctor $D$ confirms the patient $x_{r}$ does not have any symptom $y_{i}$ ), and $0 \leq \mathscr{T}_{\tilde{\mathscr{C}}_{i}}\left(x_{r}\right)+\mathscr{I}_{\widetilde{\mathscr{C}}_{i}}\left(x_{r}\right)+\mathscr{F}_{\tilde{\mathscr{C}}_{i}}\left(x_{r}\right) \leq 3$. According to the presented covering methods, we propose a decision-making algorithm to obtain the result by the following steps:
Step 1: consider, for each $x_{r} \in \Omega$, there is at least one $y_{i} \in V$ such that the symptom value $\mathscr{C}_{i}$ for patient $x_{r}$ is not less than $\beta$, where $\beta$ is a critical value.

Step 2: consider $\mathscr{A}\left(x_{r}\right)=\langle d, e, f\rangle$ is the evaluation by a decision maker $D$, where $d$ is a possible degree, $e$ is an indeterminacy degree, and $f$ is an impossible degree of $A$ disease.

Step 3: based on this information, use Definition 8 and 3-1-SVN $\beta$ CRSs model to calculate the lower and upper approximation of $\mathscr{A}$.

Step 4: calculate $\mathfrak{R}_{\mathscr{A}}$ by the following equation:

$$
\Re_{\mathscr{A}}=\mathscr{U}_{3}^{1}(\mathscr{A}) \oplus \mathscr{L}_{3}^{1}(\mathscr{A})
$$

where $\quad \mathscr{A} \oplus \mathscr{B}=\left\{\left\langle x, \mathscr{T}_{\mathscr{A}}(x)+\mathscr{T}_{\mathscr{B}}(x)-\mathscr{T}_{\mathscr{A}}(x)^{*}\right.\right.$ $\left.\left.\mathscr{T}_{\mathscr{B}}(x), \mathscr{I}_{\mathscr{A}}(x)^{*} \mathscr{I}_{\mathscr{A} B}(x), \mathscr{F}_{\mathscr{A}}(x)^{*} \mathscr{F}_{\mathscr{B}}(x)\right\rangle: x \in \Omega\right\}$.

Step 5: calculate the decision method by the following formula:

$$
\mathcal{S}(x)=\frac{\mathscr{T}_{\mathfrak{R}_{\mathscr{A}}}(x)}{\sqrt{\left(\mathscr{T}_{\mathfrak{R}_{\mathscr{d}}}(x)\right)^{2}+\left(\mathscr{I}_{\mathfrak{R}_{\mathscr{d}}}(x)\right)^{2}+\left(\mathscr{F}_{\mathfrak{R}_{\mathscr{A}}}(x)\right)^{2}}},
$$

hence, ranking the alternatives.

Based on these steps, we give an algorithm to solve the decision-making problems based on Definition 8. The steps corresponding to it are summarized in Algorithm 1.

5.1.2. Method II. Suppose that $\Omega=\left\{x_{r}: r=1, \ldots, k\right\}$ is the set of alternatives (papers), $m$ is main attributes (symptoms) (e.g., spot and steak) $V=\left\{y_{i}: i=1,2, \ldots, m\right\}$ of $A$ paper trouble, $\tilde{\mathscr{C}}_{i}\left(x_{r}\right)=\left\langle\mathscr{T}_{\tilde{C}_{i}}\left(x_{r}\right), \mathscr{I}_{\tilde{\mathscr{C}}_{i}}\left(x_{r}\right), \mathscr{F}_{\tilde{\mathscr{C}}_{i}}\left(x_{r}\right)\right\rangle$ indicates the symptom value for each paper which known by an investigator $I$, for some $\beta=\langle a, b, c\rangle$, and $(\Omega, \widetilde{\Gamma})$ is a Type-2 SVN $\beta$-CRS, where $\mathscr{T}_{\tilde{C}_{i}}\left(x_{r}\right) \in[0,1]$ (i.e., the degree that the investigator $I$ asserts the paper $x_{r}$ has symptom $y_{i}$ ), $\mathscr{I}_{\tilde{C}_{i}}\left(x_{r}\right) \in[0,1]$ (i.e., the degree that the investigator $I$ is not sure whether the paper $x_{r}$ has symptom $\left.y_{i}\right), \mathscr{F}_{\mathscr{C}_{i}}\left(x_{r}\right) \in[0,1]$ (i.e., the degree that investigator $I$ affirms paper $x_{r}$ does not have any symptom $\left.y_{i}\right)$, and $0 \leq \mathscr{T}_{\widetilde{\mathscr{C}}_{i}}\left(x_{r}\right)+\mathscr{I}_{\widetilde{\mathscr{C}}_{i}}\left(x_{r}\right)+$ 
Input: SVN decision information system $(\Omega, \widetilde{\Gamma}, \beta, \mathscr{A})$.

Output: Decision-making.

(1) Enter $\mathscr{A}, \beta$ and $\Omega$.

(2) From Definition 5, compute the 1 -SVN $\beta$-neighborhood ${ }_{1} \tilde{\mathcal{N}}_{x}^{\beta}$.

(3) From Step 2 and by Definition 7, compute 1-SVN complementary $\beta$-neighborhood $\widetilde{\mathscr{M}}_{x}^{\beta}$.

(4) From Steps 2 and 3 and by Definition 8, compute 3 -1-SVN $\beta$ CRSs $\mathscr{L}_{3}^{1}(\mathscr{A})$ and $\mathscr{U}_{3}^{1}(\mathscr{A})$

(5) Compute $\mathfrak{R}_{\mathscr{A}}$.

(6) Compute the cosine similarity measure $\delta(x)$.

(7) Obtain the decision.

Algorithm 1: Algorithm for a 1-SVN $\beta$ CRSs to make a decision.

Input: SVN decision information system $(\Omega, \widetilde{\Gamma}, \beta, \mathscr{A})$.

Output: Decision-making.

(1) Enter $\mathscr{A}, \beta$, and $\Omega$.

(2) From Definition 5, compute the 2-SVN $\beta$-neighborhood $\widetilde{N}^{\beta}$

(3) From Step 2 and by Definition 9, compute the 2-SVN complementary $\beta$-neighborhood $\widetilde{\mathscr{M}}_{x}^{\beta}$.

(4) From Steps 2 and 3 and by Definition 10, compute 3-2-SVN $\beta$ CRSs $\mathscr{L}_{3}^{2}(\mathscr{A})$ and $\mathscr{U}_{3}^{2}(\mathscr{A})$

(5) Compute $\mathfrak{R}_{\mathscr{A}}$.

(6) Compute the cosine similarity measure $\delta(x)$.

(7) Obtain the decision.

Algorithm 2: Algorithm for 2-SVN $\beta$ CRSs to make a decision.

$\mathscr{F} \tilde{C}_{i}\left(x_{r}\right) \leq 3$. According to the presented covering methods, we propose a decision-making algorithm to obtain the result by the following steps:

Step 1: consider, for each $x_{r} \in \Omega$, there is at least one $y_{i} \in V$ such that the symptom value $\mathscr{C}_{i}$ for paper $x_{r}$ is not less than $\beta$ (i.e., $\widetilde{C}_{i}(x) \geqslant \beta$ ), where $\beta$ is a critical value.

Step 2: consider $\mathscr{A}\left(x_{r}\right)=\langle d, e, f\rangle$ is the evaluation by a decision maker $I$, where $d$ is a possible degree, $e$ is an indeterminacy degree, and $f$ is an impossible degree of $A$ disease.

Step 3: based on this information, use Definition 10 and 3-2-SVN $\beta$ CRSs model to calculate the lower and upper approximation of $\mathscr{A}$.

Step 4: calculate $\mathfrak{R}_{\mathscr{A}}$ by the following equation:

$$
\mathfrak{R}_{\mathscr{A}}=\mathscr{U}_{3}^{2}(\mathscr{A}) \oplus \mathscr{L}_{3}^{2}(\mathscr{A})
$$

where $\quad \mathscr{A} \oplus \mathscr{B}=\left\{\left\langle x, \mathscr{T}_{\mathscr{A}}(x)+\mathscr{T}_{\mathscr{B}}(x)-\mathscr{T}_{\mathscr{A}}(x)^{*}\right.\right.$ $\left.\left.\mathscr{T}_{\mathscr{B}}(x), \mathscr{I}_{\mathscr{A}}(x)^{*} \mathscr{I}_{\mathscr{B}}(x), \mathscr{F}_{\mathscr{A}}(x)^{*} \mathscr{F}_{\mathscr{B}}(x)\right\rangle: x \in \Omega\right\}$.

Step 5: calculate the decision method by the following formula.

$$
\mathcal{S}(x)=\frac{\mathscr{T}_{\mathfrak{R}_{\mathscr{A}}}(x)}{\sqrt{\left(\mathscr{T}_{\mathfrak{R}_{\mathscr{A}}}(x)\right)^{2}+\left(\mathscr{I}_{\mathfrak{R}_{\mathscr{d}}}(x)\right)^{2}+\left(\mathscr{F}_{\mathfrak{R}_{\mathscr{d}}}(x)\right)^{2}}}
$$

hence, ranking the alternatives.

Based on these steps, we give an algorithm to solve the decision-making problems based on Definition 10. The steps corresponding to it are summarized in Algorithm 2.

\subsection{Numerical Example}

Example 5. Diseased people form a set $\Omega=$ $\left\{x_{1}, x_{2}, x_{3}, x_{4}, x_{5}\right\}$ and their relevant symptoms are collected by the attribute set $V=\left\{\operatorname{cough}\left(y_{1}\right)\right.$, fever $\left(y_{2}\right)$, sore $\left(y_{3}\right)$, headache $\left.\left(y_{4}\right)\right\}$ for $A$ disease. Here, the following steps of the algorithm described are implemented.

Step 1: under the attribute set, doctor $D$ estimates each patient and presents its decisions with suitable values which are summarized in Table 1.

Step 2: consider $\beta=\langle 0.5,0.3,0.8\rangle$ is a critical and $\widetilde{\Gamma}=$ $\left\{\widetilde{C}_{1}, \widetilde{C}_{2}, \widetilde{C}_{3}, \widetilde{C}_{4}\right\}$ is a Type-1 SVN $\beta$ CRS. Then, we compute the Type-1 SVN $\beta$-neighborhood ${ }_{1} \widetilde{N}_{x}^{\beta}$ and the Type-1 SVN complementary $\beta$-neighborhood ${ }_{1} \widetilde{\mathscr{M}}_{x}^{\beta}$, as shown in Tables 2 and 3.

Consider $\mathscr{A}=\left((0.6,0.3,0.5) / x_{1}\right)+((0.4,0.5,0.1) /$ $\left.x_{2}\right)+\left((0.3,0.2,0.6) / x_{3}\right)+\left((0.5,0.3,0.4) / x_{4}\right)+((0.7$, $\left.0.2,0.3) / x_{5}\right)$.

Step 3: by Definition 8 and 3-1-SVN $\beta$ RSs model, we have the following results: 


$$
\begin{aligned}
& \mathscr{L}_{3}^{1}(\mathscr{A})=\left\{\left\langle x_{1}, 0.6,0.3,0.5\right\rangle,\left\langle x_{2}, 0.6,0.3,0.4\right\rangle,\left\langle x_{3}, 0.5,0.3,0.4\right\rangle,\left\langle x_{4}, 0.4,0.2,0.3\right\rangle,\left\langle x_{5}, 0.6,0.3,0.3\right\rangle\right\}, \\
& \mathcal{U}_{3}^{1}(\mathscr{A})=\left\{\left\langle x_{1}, 0.5,0.5,0.6\right\rangle,\left\langle x_{2}, 0.4,0.5,0.6\right\rangle,\left\langle x_{3}, 0.5,0.5,0.5\right\rangle,\left\langle x_{4}, 0.5,0.5,0.6\right\rangle,\left\langle x_{5}, 0.5,0.5,0.6\right\rangle\right\} .
\end{aligned}
$$

Step 4: compute $\mathfrak{R}_{\mathscr{A}}$ as follows:

$\mathfrak{R}_{\mathscr{A}}=\mathscr{L}_{3}^{1}(\mathscr{A}) \oplus \mathcal{U}_{3}^{1}(\mathscr{A})=\left\{\left\langle x_{1}, 0.8,0.15,0.3\right\rangle,\left\langle x_{2}, 0.76,0.15,0.24\right\rangle,\left\langle x_{3}, 0.75,0.15,0.2\right\rangle,\left\langle x_{4}, 0.7,0.1,0.18\right\rangle,\left\langle x_{5}, 0.8,0.15,0.18\right\rangle\right\}$.

Step 5: according to the above information, we get $\mathcal{S}(x)$ as follows:

$$
\begin{aligned}
& \mathcal{S}\left(x_{1}\right)=0.923 \\
& \mathcal{S}\left(x_{2}\right)=0.938 \\
& \mathcal{S}\left(x_{3}\right)=0.949 \\
& \mathcal{S}\left(x_{4}\right)=0.959 \\
& \mathcal{S}\left(x_{5}\right)=0.964
\end{aligned}
$$

and hence, we get the ranking order as

$$
\mathcal{S}\left(x_{5}\right)>\mathcal{S}\left(x_{4}\right)>\mathcal{S}\left(x_{3}\right)>\mathcal{S}\left(x_{2}\right)>\mathcal{S}\left(x_{1}\right) .
$$

So, by the above computation, the verdict of the decision maker $D$ is $x_{5}$.

Example 6. Let $\Omega=\left\{x_{1}, x_{2}, x_{3}, x_{4}, x_{5}\right\}$ be the set of papers, and their relevant symptoms are collected by the attribute set
$V=\left\{\operatorname{spot}\left(y_{1}\right), \operatorname{steak}\left(y_{2}\right), \operatorname{crater}\left(y_{3}\right)\right.$, fracture $\left.\left(y_{4}\right)\right\}$ for $A$ paper error. Here, the following steps of the algorithm described are implemented.

Step 1: under the attribute set, investigator $I$ estimates each paper and presents its decisions with suitable values which are summarized in Table 1.

Step 2: consider $\beta=\langle 0.5,0.1,0.8\rangle$ is a critical and $\widetilde{\Gamma}=$ $\left\{\widetilde{C}_{1}, \widetilde{C}_{2}, \widetilde{C}_{3}, \widetilde{C}_{4}\right\}$ is a 2 -SVN $\beta$ CRS. Then, we compute the Type-2 SVN $\beta$-neighborhood ${ }_{2} \widetilde{N}_{x}^{\beta}$ and the Type-2 SVN complementary $\beta$-neighborhood ${ }_{2} \widetilde{M}_{x}^{\beta}$, as shown in Tables 6 and 7. Consider $\mathscr{A}=\left((0.6,0.3,0.5) / x_{1}\right)+$ $\left((0.4,0.5,0.1) / x_{2}\right)+\left((0.3,0.2,0.6) / x_{3}\right)+((0.5,0.3,0.4)$ $\left./ x_{4}\right)+\left((0.7,0.2,0.3) / x_{5}\right)$.

Step 3: by Definition 10 and 3-2-SVN $\beta$ RSs model, we have the following results:

$$
\begin{aligned}
& \mathscr{L}_{3}^{2}(\mathscr{A})=\left\{\left\langle x_{1}, 0.6,0.7,0.5\right\rangle,\left\langle x_{2}, 0.6,0.7,0.4\right\rangle,\left\langle x_{3}, 0.5,0.7,0.4\right\rangle,\left\langle x_{4}, 0.4,0.7,0.3\right\rangle,\left\langle x_{5}, 0.6,0.7,0.3\right\rangle\right\}, \\
& \mathcal{U}_{3}^{2}(\mathscr{A})=\left\{\left\langle x_{1}, 0.5,0.2,0.6\right\rangle,\left\langle x_{2}, 0.4,0.2,0.6\right\rangle,\left\langle x_{3}, 0.5,0.3,0.5\right\rangle,\left\langle x_{4}, 0.5,0.2,0.6\right\rangle,\left\langle x_{5}, 0.5,0.3,0.6\right\rangle\right\} .
\end{aligned}
$$

Step 4: compute $\mathfrak{R}_{\mathscr{A}}$ as follows:

$\Re_{\mathscr{A}}=\mathscr{L}_{3}^{2}(\mathscr{A}) \oplus \mathcal{U}_{3}^{2}(\mathscr{A})=\left\{\left\langle x_{1}, 0.8,0.14,0.3\right\rangle,\left\langle x_{2}, 0.76,0.14,0.24\right\rangle,\left\langle x_{3}, 0.75,0.21,0.2\right\rangle,\left\langle x_{4}, 0.7,0.14,0.18\right\rangle,\left\langle x_{5}, 0.8,0.21,0.18\right\rangle\right\}$.

Step 5: according to above information, we get $\mathcal{S}(x)$ as follows:

$$
\begin{aligned}
& \mathcal{S}\left(x_{1}\right)=0.924 \\
& \mathcal{S}\left(x_{2}\right)=0.939 \\
& \mathcal{S}\left(x_{3}\right)=0.933 \\
& \mathcal{S}\left(x_{4}\right)=0.951 \\
& \mathcal{S}\left(x_{5}\right)=0.945
\end{aligned}
$$

and hence, we get the ranking order as

$$
\mathcal{S}\left(x_{4}\right)>\delta\left(x_{5}\right)>\delta\left(x_{2}\right)>\delta\left(x_{3}\right)>\delta\left(x_{1}\right)
$$

So, by the above calculations, the verdict of the decision maker $I$ is $x_{4}$.

5.3. Comparative Analysis. The major purpose of our presented work is eligible to raise the lower approximation and reduce the upper approximation of the previous study by Wang and Zhang's methods [46, 47], as visible in Examples 2 and 4. To clarify the comparisons between Wang and Zhang's methods [46, 47] and our methods, the sorting outcomes of these decision-making models are listed in Table 10 for 1 -SVN $\beta$ CAS and Table 11 for 2-SVN $\beta$ CAS. 
TABLE 10: Sorting outcomes for 1-SVN $\beta$ CAS.

\begin{tabular}{ll}
\hline Different methods & Obtain a decision \\
\hline Wang and Zhang's model [46] & $x_{5}>x_{1}>x_{2}>x_{3}>x_{4}$ \\
Our model & $x_{5}>x_{4}>x_{3}>x_{2}>x_{1}$ \\
\hline
\end{tabular}

TABLE 11: Sorting outcomes for 2-SVN $\beta$ CAS.

\begin{tabular}{ll}
\hline Different methods & Obtain a decision \\
\hline Wang and Zhang's model [47] & $x_{5}>x_{1}>x_{2}>x_{4}>x_{3}$ \\
Our model & $x_{4}>x_{5}>x_{2}>x_{3}>x_{1}$ \\
\hline
\end{tabular}

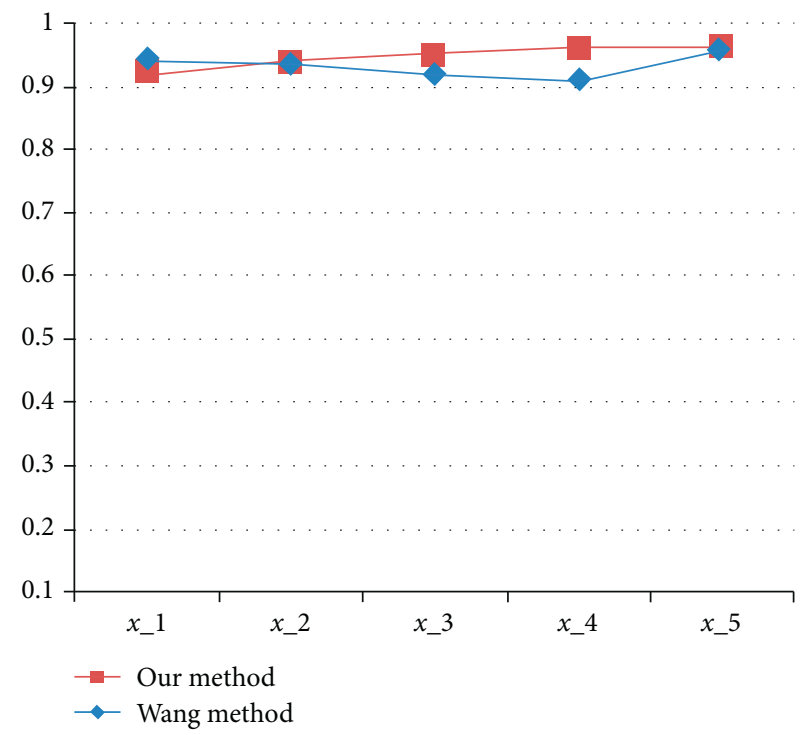

FIGURE 1: The representations of the results by using our model and Wang and Zhang's model [46].

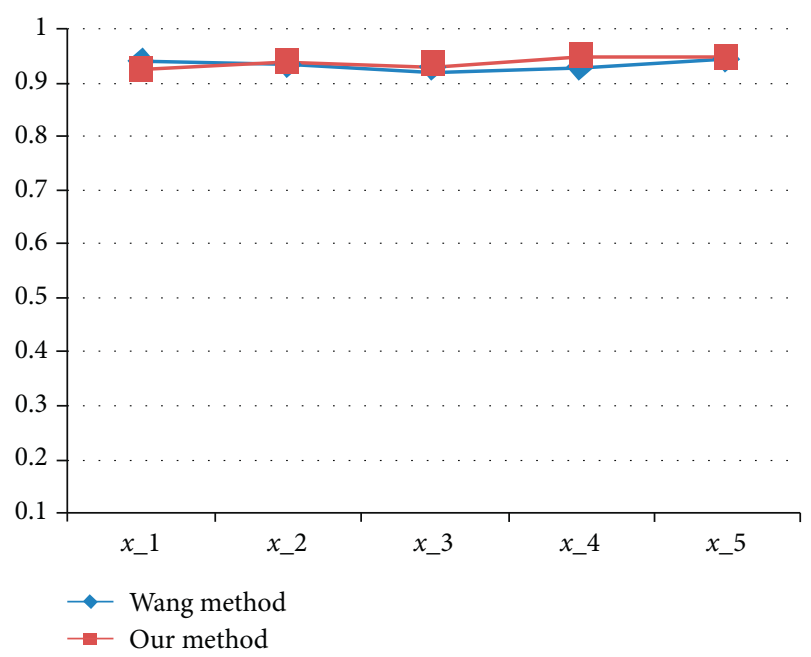

FIGURE 2: The representations of the results by using our model and Wang and Zhang's model [47].
An easy way to explain these outcomes, see Figures 1 and 2 which simplify the comparisons between our presented method and the previous one.

Figure 1 explained the differences between the outcomes using our model (3-1-SVN $\beta$ CAS) and the last one (1-1SVN $\beta$ CRSs). Furthermore, Figure 2 illustrated the comparisons between the values through our model (3-2SVN $\beta$ CAS) and the previous one (1-2-SVN $\beta$ CRSs). Thus, there are slight differences among these distinct methods, and these variations made our model better than others.

\section{Conclusion}

This work is extended to Wang and Zhang's studies in $[46,47]$. We presented the definitions of 1-SVN complementary $\beta$-neighborhoods and 2-SVN complementary $\beta$-neighborhoods. We use them to set up new models of 1 -SVN $\beta$-CRS and 2 -SVN $\beta$-CRS, respectively. Moreover, by merging the Type-1 neighborhoods (resp., Type-2 neighborhoods) and Type-1 complementary neighborhoods (resp., Type-2 complementary neighborhoods), we obtain two new types of Type-1 neighborhoods and Type-2 neighborhoods, respectively. Thus, two new classes of 1-SVN $\beta$-CRS and 2 -SVN $\beta$-CRS are investigated. To explain the differences between these new and older types of covering methods, see Examples 2 and 4. For more clarification about them, see Figures 1 and 2. There are some issues in these two covering methods:

(1) If $\beta=(0.5,0.1,0.8)$ in Example 2 , then $\widetilde{\Gamma}$ is not 1 SVN $\beta$ CRSs, but it is applicable in 2-SVN $\beta$ CRSs

(2) If $\beta=(0.5,0.3,0.8)$ in Example 4 , then $\widetilde{\Gamma}$ is not 2 SVN $\beta$ CRSs, but it is applicable in 1-SVN $\beta$ CRSs

In short, the two methods are considered complementary to each other, which means if there are some failures in 1 -SVN $\beta$ CRSs, the 2 -SVN $\beta$ CRSs is working instead and vice versa.

In the future, we can extend the results of this study as a combination between 1-SVN (or 2-SVN) complementary $\beta$-neighborhoods and published papers (see [50-55]). In addition, one may investigate further based on 1-SVN (or 2-SVN) complementary $\beta$-neighborhoods with some links to topology as in $[26,48]$. Finally, there are many areas (for example, several comparative of this proposed method) which can be presented by researchers in the next paper.

\section{Data Availability}

No data were used to support this study.

\section{Conflicts of Interest}

The authors declare that they have no conflicts of interest.

\section{Acknowledgments}

This study was supported by the Science and Technology Project of Yulin City (CXY-2020-007). 


\section{References}

[1] Z. Pawlak, "Rough sets," International Journal of Computer \& Information Sciences, vol. 11, no. 5, pp. 341-356, 1982.

[2] Z. Pawlak, "Rough concept analysis," Bulletin of the Polish Academy of Sciences Mathematics, vol. 33, pp. 9-10, 1985.

[3] M. Atef, A. M. Khalil, S.-G. Li, A. A. Azzam, and A. E. F. El Atik, "Comparison of six types of rough approximations based on $\mathrm{j}$-neighborhood space and $\mathrm{j}$-adhesion neighborhood space," Journal of Intelligent \& Fuzzy Systems, vol. 39, no. 3, pp. 4515-4531, 2020.

[4] J. C. R. Alcantud and J. Zhan, "Multi-granular soft rough covering sets," Soft Computing, vol. 24, no. 13, pp. 9391-9402, 2020.

[5] A. A. El Atik, A. S. Nawar, and M. Atef, "Rough approximation models via graphs based on neighborhood systems," Granular Computing, 2020.

[6] T. Herawan, M. M. Deris, and J. H. Abawajy, "A rough set approach for selecting clustering attribute," Knowledge-Based Systems, vol. 23, no. 3, pp. 220-231, 2010.

[7] Q. Hu, L. Zhang, D. Chen, W. Pedrycz, and D. Yu, "Gaussian kernel based fuzzy rough sets: model, uncertainty measures and applications," International Journal of Approximate Reasoning, vol. 51, no. 4, pp. 453-471, 2010.

[8] K. Y. Huang, T.-H. Chang, and T.-C. Chang, "Determination of the threshold value $\beta$ of variable precision rough set by fuzzy algorithms $\beta$ of variable precision rough set by fuzzy algorithms," International Journal of Approximate Reasoning, vol. 52, no. 7, pp. 1056-1072, 2011.

[9] R. Jensen and Q. Shen, "Semantics-preserving dimensionality reduction: rough and fuzzy-rough-based approaches," IEEE Transactions on Knowledge and Data Engineering, vol. 16, no. 12, pp. 1457-1471, 2004.

[10] G. Liu and W. Zhu, "The algebraic structures of generalized rough set theory," Information Sciences, vol. 178, no. 21, pp. 4105-4113, 2008.

[11] S. Pal and P. Mitra, "Case generation using rough sets with fuzzy representation," IEEE Transactions on Knowledge and Data Engineering, vol. 16, pp. 293-300, 2004.

[12] Y. Qian, J. Liang, and C. Dang, "Knowledge structure, knowledge granulation and knowledge distance in a knowledge base," International Journal of Approximate Reasoning, vol. 50, no. 1, pp. 174-188, 2009.

[13] X. Yang and T. Li, "The minimization of axiom sets characterizing generalized approximation operators," Information Sciences, vol. 176, no. 7, pp. 887-899, 2006.

[14] Y. Yao, "Three-way decisions with probabilistic rough sets," Information Sciences, vol. 180, no. 3, pp. 341-353, 2010.

[15] H. Zhang, H. Liang, and D. Liu, "Two new operators in rough set theory with applications to fuzzy sets," Information Sciences, vol. 166, no. 1-4, pp. 147-165, 2004.

[16] B. Sun, W. Ma, and Y. Qian, "Multigranulation fuzzy rough set over two universes and its application to decision making," Knowledge-Based Systems, vol. 123, pp. 61-74, 2017.

[17] W. Wu and W. X. Zhang, "Constructive and axiomatic approaches of fuzzy approximation operators," Information Sciences, vol. 159, no. 3-4, pp. 233-254, 2004.

[18] D. S. Yeung, D. Chen, J. Lee, and X. Wang, "On the generalization of fuzzy rough sets," IEEE Transaction Fuzzy System, vol. 13, pp. 343-361, 2015.

[19] W. Ziarko, "Variable precision rough set model," Journal of Computer and System Sciences, vol. 46, no. 1, pp. 39-59, 1993.

[20] J. A. Pomykala, "Approximation operations in approximation space," Bulletin of the Polish Academy of Science, vol. 35, pp. 653-662, 1987.
[21] J. A. Pomykala, "On definability in the nondeterministic information system," Bulletin of the Polish Academy of Science, vol. 36, pp. 193-210, 1988.

[22] Y. Y. Yao, "Relational interpretations of neighborhood operators and rough set approximation operators," Information Sciences, vol. 111, no. 1-4, pp. 239-259, 1998.

[23] Y. Yao and B. Yao, "Covering based rough set approximations," Information Sciences, vol. 200, pp. 91-107, 2012.

[24] I. Couso and D. Dubois, "Rough sets, coverings and incomplete information," Fundamenta Informaticae, vol. 108, no. 3-4, pp. 223-247, 2011.

[25] Z. Bonikowski, E. Bryniarski, and U. Wybraniec-Skardowska, "Extensions and intentions in the rough set theory," Information Sciences, vol. 107, no. 1-4, pp. 149-167, 1998.

[26] W. Zhu, "Topological approaches to covering rough sets," Information Sciences, vol. 177, no. 6, pp. 1499-1508, 2007.

[27] W. Zhu and F.-Y. Wang, "Reduction and axiomization of covering generalized rough sets," Information Sciences, vol. 152, pp. 217-230, 2003.

[28] W. Zhu and F.-Y. Wang, "On three types of covering-based rough sets," IEEE Transactions on Knowledge and Data Engineering, vol. 19, no. 8, pp. 1131-1144, 2007.

[29] W. Zhu and F.-Y. Wang, "The fourth type of covering-based rough sets," Information Sciences, vol. 201, pp. 80-92, 2012.

[30] E. C. C. Tsang, C. Degang, and D. S. Yeung, "Approximations and reducts with covering generalized rough sets," Computers \& Mathematics with Applications, vol. 56, no. 1, pp. 279-289, 2008.

[31] W.-H. Xu and W.-X. Zhang, "Measuring roughness of generalized rough sets induced by a covering," Fuzzy Sets and Systems, vol. 158, no. 22, pp. 2443-2455, 2007.

[32] G. Liu and Y. Sai, "A comparison of two types of rough sets induced by coverings," International Journal of Approximate Reasoning, vol. 50, no. 3, pp. 521-528, 2009.

[33] L. Ma, "On some types of neighborhood-related covering rough sets," International Journal of Approximate Reasoning, vol. 53, no. 6, pp. 901-911, 2012.

[34] D. Dubois and H. Prade, "Rough fuzzy sets and fuzzy rough sets," International Journal of General Systems, vol. 17, no. 2-3, pp. 191-209, 1990.

[35] L. A. Zadeh, "Fuzzy sets," Information and Control, vol. 8, no. 3, pp. 338-353, 1965.

[36] L. Ma, "Two fuzzy covering rough set models and their generalizations over fuzzy lattices," Fuzzy Sets and Systems, vol. 294, pp. 1-17, 2016.

[37] B. Yang and B. Q. Hu, "On some types of fuzzy covering-based rough sets," Fuzzy Sets and Systems, vol. 312, pp. 36-65, 2017.

[38] B. Yang and B. Q. Hu, "Fuzzy neighborhood operators and derived fuzzy coverings," Fuzzy Sets and Systems, vol. 370, pp. 1-33, 2019.

[39] H. Wang, F. Smarandache, Y. Zhang, and R. Sunderraman, "Single valued neutrosophic sets," Multispace Multistruct, vol. 4, pp. 410-413, 2010.

[40] K. T. Atanassov, "Intuitionistic fuzzy sets," Fuzzy Sets and Systems, vol. 20, no. 1, pp. 87-96, 1986.

[41] F. Smarandache, Neutrosophy, Neutrosophic Probability, Set, and Logic, American Research Press, Santa Fe, NM, USA, 1998.

[42] I. B. Turksen, "Interval valued fuzzy sets based on normal forms," Fuzzy Sets and Systems, vol. 20, no. 2, pp. 191-210, 1986.

[43] K. Atanassov and G. Gargov, "Interval valued intuitionistic fuzzy sets," Fuzzy Sets and Systems, vol. 31, no. 3, pp. 343-349, 1989. 
[44] K. Mondal and S. Pramanik, "Rough neutrosophic multiattribute decision-making based on grey relational analysis," Neutrosophic Sets and Systems, vol. 7, pp. 8-17, 2015.

[45] H.-L. Yang, C.-L. Zhang, Z.-L. Guo, Y.-L. Liu, and X. Liao, “A hybrid model of single valued neutrosophic sets and rough sets: single valued neutrosophic rough set model," Soft Computing, vol. 21, no. 21, pp. 6253-6267, 2017.

[46] J. Wang and X. Zhang, "Two types of single valued neutrosophic covering rough sets and an application to decision making," Symmetry, vol. 10, no. 12, p. 710, 2018.

[47] J. Wang and X. Zhang, "A new type of single valued neutrosophic covering rough set model," Symmetry, vol. 11, no. 9, p. 1074, 2019.

[48] M. Riaz, F. Smarandache, F. Karaaslan, M. R. Hashmi, and I. Nawaz, "Neutrosophic soft rough topology and its applications to multi-criteria decision-making," Neutrosophic Sets and Systems, vol. 35, pp. 198-218, 2020.

[49] A. Al-Quran, N. Hassan, and E. Marei, "A novel approach to neutrosophic soft rough set under uncertainty," Symmetry, vol. 11, no. 3, p. 384, 2019.

[50] J. Wang and X. Zhang, "Multigranulation single valued neutrosophic covering-based rough sets and their applications to multi-criteria group decision making," Iranian Journal of Fuzzy Systems, vol. 17, no. 5, pp. 109-126, 2020.

[51] S. Broumi, A. Bakali, M. Talea et al., "Bipolar complex neutrosophic sets and its application in decision making problem," in Fuzzy Multi-Criteria Decision-Making Using Neutrosophic Sets, pp. 677-710, Springer, Cham, Switzerland, 2019.

[52] N. Hassan, V. Uluçay, and M. Şahin, "Q-neutrosophic soft expert set and its application in decision making," International Journal of Fuzzy System Applications, vol. 7, no. 4, pp. 37-61, 2018.

[53] V. Uluçay, M. Şahin, and N. Hassan, "Generalized neutrosophic soft expert set for multiple-criteria decision-making," Symmetry, vol. 10, no. 10, p. 437, 2018.

[54] T. Feng, S.-P. Zhang, and J.-S. Mi, "The reduction and fusion of fuzzy covering systems based on the evidence theory," International Journal of Approximate Reasoning, vol. 53, no. 1 , pp. 87-103, 2012.

[55] T.-J. Li, Y. Leung, and W.-X. Zhang, "Generalized fuzzy rough approximation operators based on fuzzy coverings," International Journal of Approximate Reasoning, vol. 48, no. 3, pp. 836-856, 2008. 